\title{
Evolutionary dynamics in markets with many trader types
}

\author{
William A. Brock \\ Department of Economics, University of Wisconsin \\ Cars H. Hommes \\ Florian O. O. Wagener \\ Department of Quantitative Economics, CeNDEF, University of Amsterdam
}

July 10, 2003

Submitted to

the Special Issue of the Journal of Mathematical Economics on

Evolutionary Finance

\begin{abstract}
This paper develops the notion of a Large Type Limit (LTL) describing the dynamical behavior of heterogeneous markets with many trader types. It is shown that generic and persistent features of adaptive evolutionary systems with many trader types are well described by the large type limit. Stability and bifurcation routes to instability and strange attractors in a simple evolutionary financial market model are studied. An increase in the "intensity of adaption" or in the diversity of beliefs may lead to deviations from an unstable RE fundamental benchmark and excess volatility. A large evolutionary system may thus become unstable and complicated dynamics may arise when agents become sensitive to small differences in fitness.
\end{abstract}

JEL classification: E32, G12, D84

Keywords: large multi-agent system, evolutionary learning, bounded rationality, bifurcation and chaos

Acknowledgments. This work was presented at the Evolutionary Finance conference at Zürich, Switzerland, June 7-8, 2002. Earlier versions of the paper were presented at the IFAC symposium Computational Economics, Finance and Engineering, Cambridge, June 29-July 1, 1998, the CeNDEF workshop on Economic Dynamics, 
Amsterdam, January 13-15, 2000, the 7th Viennese Workshop on Optimal Control, Dynamic Games and Nonlinear Dynamics: Theory and Applications in Economics and OR/MS, Vienna, May 24-26, 2000 and the IFAC symposium Computational Economics, Finance and Engineering, Yale, June 28-30, 2001. Stimulating discussions with participants are gratefully acknowledged. We also would like to thank an anonymous referee and the editors of this special issue, Klaus-Reiner Schenk-Hoppé and Thorsten Hens for helpful comments on an earlier draft. This research has been supported by the Netherlands Organization for Scientific Research (NWO) under a NWO-MaG Pionier grant. W.A. Brock thanks the NSF under Grant \#SES-9911251 and the Vilas Trust for essential support. None of the above are responsible for errors in this paper.

Corresponding author: Florian Wagener, Center for Nonlinear Dynamics in Economics and Finance (CeNDEF), University of Amsterdam, Roetersstraat 11, NL-1018 WB Amsterdam, The Netherlands; e-mail: f.o.o.wagener@uva.nl; homepage: http://www.fee.uva.nl/cendef/ 


\section{Contents}

1 Introduction 1

2 Large Type Limit 5

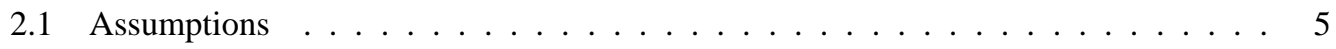

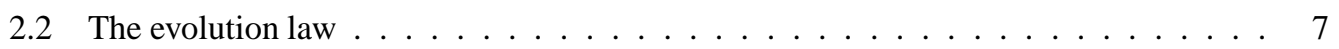

2.3 Statement of the theorem $\ldots \ldots \ldots \ldots \ldots \ldots$

3 Dynamical consequences $\quad 9$

3.1 Structural stability and persistence $\ldots \ldots \ldots \ldots \ldots \ldots$

3.2 Application to large type limits . . . . . . . . . . . . . . . . 12

4 A financial market with heterogeneous beliefs $\quad 15$

4.1 The asset pricing model with heterogeneous beliefs $\ldots \ldots \ldots \ldots$

4.2 The Large Type Limit of the asset pricing model . . . . . . . . . . . . . . . . 21

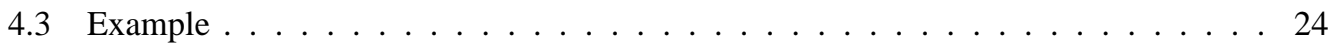

5 Concluding Remarks and Future Research 28 


\section{Introduction}

Do expectations matter or do asset prices fully reflect economic fundamentals? Do heterogeneous beliefs of millions of different investors cancel out on average due to aggregation, or can optimistic or pessimistic views cluster together and cause prices to deviate from underlying economic fundamentals? These questions have been a matter of heavy debate among economists as well as financial practitioners for many decades already. Keynes, for example, argued that investment based on long term expectations of market fundamentals is risky and that it might be more reasonable "to guess better than the crowd how the crowd will behave" (Keynes, 1936, p.157). In contrast, new classical economists have viewed "market psychology" and "investors sentiment" as being irrational and therefore inconsistent with the rational expectations hypothesis (REH) and the efficient market hypothesis (EMH). Friedman (1953), for example, argued that irrational speculative traders would be driven out of the market by rational traders, who would trade against them by taking long opposite positions, thus driving prices back to fundamentals.

In empirical work much attention has been paid to the closely related question whether asset prices exhibit excess volatility, that is, whether volatility of asset prices is larger than volatility of underlying economic fundamentals. In particular the work by Shiller $(1989,2000)$ has emphasized the possibility of excess volatility and persistent deviations of asset prices from a fundamental RE benchmark. In behavioral finance, for example, Thaler (1994) has argued that quasi-rationality may be a key source of deviations from the RE fundamental benchmark and excess volatility. Quasi rationality means less than fully rational behavior, for example, due to investors' sentiment, overconfidence or overreaction. However work by Boldrin and Levine (2001) argues that patterns of returns behavior that look like evidence of "irrationality" or "bounded rationality" may be simply rational reaction of stock markets to earnings profiles generated by technological change. Our paper is focused more on higher frequency fluctuations than Boldrin and Levine (2001), but related arguments must always make one wary of concluding that patterns of booms and crashes in stock market values are evidence of any kind of irrational pricing.

Experimental work has also addressed the question of possible deviations from the benchmark RE fundamental in speculative asset markets. For example, Smith, Suchanek and Williams (1988) showed that speculative bubbles and deviations from a benchmark RE fundamental are frequently observed in experimental asset markets. These temporary bubbles tend to disappear however towards the end of the experimental asset market and also tend to become smaller as traders become more experienced. Kleidon (1994) has written a recent review paper about market "pathologies" such as crashes, blow offs, excess volatility, anomalies, etc.; see also Plott and Sunder (1988) and Camerer 
(1989). In particular he points out how the experimental literature in an asymmetric information setting has shown that REE is not achieved immediately but tends to be achieved by experienced traders.

The debate whether expectations affect asset prices and may lead to excess volatility should be viewed in the light of two important developments in the recent literature: bounded rationality and heterogeneous agents systems. Bounded rationality and heterogeneous agents may be viewed as building blocks in behavioral finance, where traders are viewed as boundedly rational agents using simple, habitual rule of thumb rules, see e.g. Shleifer (2000) and Hirshleifer (2001) for recent surveys of behavioral finance. Although rational expectations remains an important benchmark, work on bounded rationality in the past decade may be viewed as an attempt to explore deviations from this benchmark. General surveys on bounded rationality in expectations and learning are e.g. Sargent (1993), Grandmont (1998) and Evans and Honkapohja (2001); see also Kurz's book (1997) which develops the theory of rational beliefs (RBE). In particular, Sargent (1999) argues that many of the bounded rational expectations equilibria may be viewed as 'approximate' rational expectations equilibria.

In the last decade, a rapidly increasing interest in multi-agent systems can be observed. Markets viewed as evolutionary adaptive systems with boundedly rational interacting agents have e.g. been studied in Arthur et al. (1997), Brock and Hommes (1997ab,1998), Chiarella and He (2000,2002), Farmer (1998), Gaunersdorfer and Hommes (2000), Kirman (1991), LeBaron et al. (1999), Lux (1995) and Lux and Marchesi (1999,2000). These developments in multi-agent systems are closely related to recent work in finance on 'smart money' and noise traders e.g. by Frankel and Froot (1988), De Long, Shleifer, Summers and Waldmann (1990), and Wang (1994). A common feature of these contributions is that there are two different classes of investors that can also be observed in financial practice: fundamentalist and technical analysts. Fundamentalists base their forecasts of future prices and returns upon economic fundamentals, such as dividends, interest rates, priceearning ratio's, etc. In contrast, technical analysts are looking for patterns in past prices and base their forecasts upon extrapolation of these patterns. An interesting outcome of these evolutionary heterogeneous agent systems is that the models mimic a number of stylized facts frequently observed in financial series, such as unpredictability of returns, fat tails, volatility clustering and long memory. Price deviations from the RE fundamental and excess volatility are triggered by uncertainty about economic fundamentals but may be amplified by evolutionary interaction of competing, boundedly rational trading strategies. Most of this work is computationally oriented and based upon computer simulations of complex adaptive systems.

The present paper provides a theoretical framework for evolutionary markets with many different 
trader types. We introduce the notion of a so-called Large Type Limit (LTL), which is a simple, low dimensional system describing the evolutionary dynamics in a market with many trader types. The concept of LTL was sketched in Brock (1997), Brock and Hommes (1999) and Brock and de Fontnouvelle (2000) but is actually rigorously developed in this paper ${ }^{1}$. The LTL concept will be developed within a general heterogeneous markets framework, and we will apply it to a simple evolutionary asset pricing model with heterogeneous beliefs and study its dynamical behaviour in some detail. The LTL is a type of ensemble limit rather like thermodynamic limits in statistical mechanics. It is motivated by observing that market equilibrium equations tend to have a form which is a function of expressions that look like sample moments. The LTL is then simply obtained by replacing sample moments by population moments. For particular distributions of characteristics, one may obtain closed form solutions for these population moments and thus obtain closed form expressions for objects that appeared intractable. The LTL can also be viewed as a device that effectively reduces a large number of parameters inherent in models with a large number of different trader types by introducing an underlying characteristics distribution which itself can be characterized by a small number of parameters. Thus empirical work can be assisted by this type of parameter reduction technique. We think this is a new method of analysis which is of independent interest. The LTL theory developed here can be used to form a bridge between analytical results and the literature on evolutionary simulation of asset trading, which has become very popular in the last few years (see e.g. Arthur $e t$ al. (1997), LeBaron (2000) and Hommes (2001) for reviews). In fact, one of the main results of the present paper is that the LTL well approximates the evolutionary dynamics in a market with many trader types in the sense that all generic and persistent features of an evolutionary adaptive system with many trader types are preserved in the LTL. Hence, the LTL can be used to study local stability of the benchmark RE fundamental steady state as well as possible deviations from the RE benchmark and bifurcations routes to instability and complicated periodic or even chaotic dynamics in adaptive evolutionary systems with many trader types.

Let us now discuss some closely related recent work. Brock and Hommes (1997a) introduced a tractable form of evolutionary dynamics in the cobweb demand-supply model with a costly, sophisticated forecasting rule such as rational expectations competing against a cheap habitual rule of thumb forecasting rule such as adaptive expectations. Brock and Hommes (1998), henceforth BH, called this evolutionary dynamics an Adaptive Belief System (ABS) and applied the framework to

\footnotetext{
${ }^{1}$ The notion of LTL is related to the well known measure space approach in general equilibrium theory (See Kirman (1981) and Hildenbrand (1982) for reviews). The main difference lies in locating intertemporal dynamical equilibrium relationships that resemble sample moment conditions to suggest the form of the appropriate limit and establishing which dynamical phenomena are "persistent" under "finite" economy limits, so that the limit itself can be directly used to construct bifurcation diagrams and predict the dynamical bifurcation behavior for large enough "samples." Details will follow.
} 
asset pricing theory in a model with one risky asset and one risk free asset. Tractability is achieved by the use of random utility models to model 'fitness' and 'natural selection.' Predictor choice across the set of predictors is evolutionarily rational in the sense that agents choose the predictors according to highest fitness, such as past profits. In the ABS studied by BH there were only few trader types, typically only two, three or four. The present paper generalizes the ABS to an evolutionary system with many different trader types and proposes to approximate the evolutionary dynamics with many trader types by a LTL.

An important feature of the $\mathrm{BH}$ framework is that the RE benchmark is nested within the evolutionary model as a special case. The benchmark fundamental represents the traders' response to common knowledge exogenous news, such as interest rate movements, growth of the firms profits, earnings or dividends, a repeal of the capital gains tax, etc. Traders' beliefs about future prices are formulated in terms of deviations from a benchmark RE fundamental. These deviations can be viewed as each RE trader's belief about how the deviations from RE by the rest of the trading community might show up in equilibrium prices. In this sense our theory is fully rational in the sense that truly rational traders must take into account the behavior of other traders in the trading community. The $\mathrm{BH}$ framework is motivated by the kinds of evidence reviewed above and starts the task of building a theory that "backs off" from RE in the manner of Sargent (1993), but nests RE in such a way that RE-econometric technology such as methods based upon orthogonality conditions can be readily adapted to test the "significance" of the extra "free parameters" that our theory adds to RE theory. Pro-RE economists loosely argue that the situations in which these extra free parameters are significant may not be frequent. Anti-RE types argue that such departures may be frequent for anti-RE types. We think a theory is needed that nests both views in a way such that econometric methods are suggested to test the null of RE against the alternative. We wish to contribute to the task of building a theory in which the data can speak to the controversy on RE and excess volatility. It is worthwhile noting that Baak (1999) and Chavas (2000) have run empirical tests for heterogeneity in expectations in agricultural data and indeed find evidence for the presence of boundedly rational traders in the hog and cattle markets. Recently Manzan (2003) estimated an evolutionary heterogeneous agent model on the S\&P 500 index, and found empirical evidence of the presence of trend followers, especially in the late nineties when trend following trading rules reenforced the rise in asset price.

The plan of the paper is as follows. Section 2 introduces the notion of large type limit (LTL) in a general heterogeneous market equilibrium framework and proves the LTL convergence theorem stating that when the number of trader types goes to infinity the heterogeneous market converges almost surely to its LTL. Section 3 discusses the dynamical consequences of the LTL convergence theorem. It is shown that the dynamical behaviour of heterogeneous markets with many trader types 
is well described by its large type limit, in the sense that all generic and persistent dynamical features (e.g. stability, bifurcations, strange attractors) of the LTL system occur with high probability in dynamic market systems with many trader types. In section 4 the LTL concept is applied to a simple evolutionary asset pricing model with heterogeneous beliefs and local stability of the fundamental steady state as well as bifurcation routes to instability and chaos in the evolutionary dynamics are studied.

\section{Large Type Limit}

The notion of Large Type Limit (LTL) will be developed within a general heterogeneous market equilibrium framework. Our starting point is the equilibrium equation at time $t$ for a market with heterogeneous sellers and buyers:

$$
\sum_{i=1}^{I} d_{i}(q) D_{i}(p, q)=\sum_{j=1}^{J} s_{j}(q) S_{j}(p, q) .
$$

Here $D_{i}(p, q)$ is the demand function of buyer type $i$; it depends on the current market price $p=p_{t}$, and another variable $q$, describing the generally available system information, like past prices and system parameters. To be specific, it can be assumed that for some $L \geq 1, M \geq 1$ :

$$
q=\left(p_{t-1}, \cdots, p_{t-L}, \lambda_{1}, \cdots, \lambda_{M}\right)
$$

The relative weight or fraction of buyer type $i$ in the market is given by $d_{i}(q)$ and depends upon past system information; all $d_{i}$ sum up to 1 . Likewise, $S_{j}(p, q)$ is the supply function of seller type $j$, and $s_{j}$ is the relative weight or fraction of that type.

\subsection{Assumptions}

We make the following assumptions about demand and supply functions and fractions:

A1. There are vector valued stochastic variables $\Theta^{d}$ and $\Theta^{s}$, with probability measures $\nu^{d}$ and $\nu^{s}$, such that

$$
D_{i}(p, q)=D\left(p, q, \vartheta_{i}^{d}\right), \quad d_{i}(q)=d\left(q, \vartheta_{i}^{d}\right)
$$

with $\vartheta_{i}^{d}$ a realisation of $\Theta^{d}$, and

$$
S_{j}(p, q)=S\left(p, q, \vartheta_{j}^{s}\right), \quad s_{j}(q)=s\left(q, \vartheta_{j}^{s}\right)
$$

with $\vartheta_{j}^{s}$ a realisation of $\Theta^{s}$. 
A2. There are functions $w^{d}\left(q, \vartheta_{i}^{d}\right)>0$, such that

$$
d\left(q, \vartheta_{i}^{d}\right)=\frac{w^{d}\left(q, \vartheta_{i}^{d}\right)}{\sum_{k} w^{d}\left(q, \vartheta_{k}^{d}\right)} .
$$

The value of $w^{d}\left(q, \vartheta_{i}^{d}\right)$ is the weight of buyer type $i$ at time $t$. Similarly, there are functions $w^{s}\left(q, \vartheta_{j}^{s}\right)>0$, such that

$$
s\left(q, \vartheta_{j}^{s}\right)=\frac{w^{s}\left(q, \vartheta_{j}^{s}\right)}{\sum_{k} w^{s}\left(q, \vartheta_{k}^{s}\right)} .
$$

The value of $w^{s}\left(q, \vartheta_{j}^{s}\right)$ is the weight of seller type $j$ at time $t$.

A3. Let $F(p, q, \vartheta)=F\left(p, q, \vartheta^{d}, \vartheta^{s}\right)$ denote the function

$$
F(p, q, \vartheta)=D\left(p, q, \vartheta^{d}\right)-S\left(p, q, \vartheta^{s}\right)
$$

It is assumed that, for any $\vartheta=\left(\vartheta^{d}, \vartheta^{s}\right)$,

$$
\begin{gathered}
F(0, q, \vartheta)>0, \\
F(+\infty, q, \vartheta)<0,
\end{gathered}
$$

and that there exists some $c<0$, such that

$$
\frac{\partial F}{\partial p}(p, q, \vartheta) \leq c<0 .
$$

In economic terms, this implies that if a buyer of any type meets a seller of any type, there is a unique equilibrium price.

A4. All functions are assumed to be infinitely continuously differentiable (or smooth) in $p$ and $q$, and continuous in $\vartheta^{d}$ and $\vartheta^{s}$.

Let $w(q, \vartheta)=w^{d}\left(q, \vartheta^{d}\right) w^{s}\left(q, \vartheta^{s}\right)$, and let for any multi-index $\alpha=\left(\alpha_{0}, \cdots, \alpha_{L+M}\right)$ the expression $D_{\alpha} F$ denote the following derivative of $F$ with respect to $p$ and $q$ :

$$
D_{\alpha} F=\frac{\partial^{\alpha_{0}}}{\partial p^{\alpha_{0}}} \frac{\partial^{\alpha_{1}}}{\partial q_{1}^{\alpha_{1}}} \cdots \frac{\partial^{\alpha_{L+M}}}{\partial q_{L+M}^{\alpha_{L+M}}} F(p, q, \vartheta) .
$$

It is assumed that for every $\alpha$ there exists a random variable $X_{\alpha}$, independent of $p$ and $q$, such that

$$
\left|w(q, \Theta) D_{\alpha} F(p, q, \Theta)\right| \leq X_{\alpha}, \quad \text { and } E X_{\alpha}<\infty .
$$

Assumption A1 states that heterogeneous demand and supply functions can be parameterized by stochastic parameter vectors $\vartheta_{i}^{d}$ and $\vartheta_{j}^{s}$ drawn from probability distributions $\nu^{d}$ and $\nu^{s}$. For example, in the financial market application in section 4 the demand type will be determined completely by 
the forecasting function of the future price of a risky asset, and the vector $\vartheta_{i}^{d}$ contains the stochastic coefficients of a (linear) forecasting function. According to assumption A2, the fractions are determined by positive weights $w^{d}\left(q, \vartheta_{i}^{d}\right)$ and $w^{s}\left(q, \vartheta_{j}^{s}\right)$. In an evolutionary setting, one may think of these weights as being derived from an evolutionary fitness measure based upon, for example, past realized trading profits. Assumption A3 implies that, for any combination of buyer and seller types, the corresponding excess demand function is strictly (and uniformly) decreasing and has a unique equilibrium price. This assumption is sufficient to ensure that the heterogeneous market equilibrium equation (1) always has a unique market clearing price. Assumption A4 is a technical assumption stating that all relevant functions are smooth and that $w(q, \Theta) D_{\alpha} F(p, q, \Theta)$ is uniformly Lebesgue dominated by a positive random variable $X_{\alpha}$ with finite unconditional mean.

\subsection{The evolution law}

We will now derive the evolution law of the heterogeneous agents market and the corresponding Large Type Limit evolution law. From assumptions A1 and A2 above, it follows that equation (1) can be rewritten as

$$
\sum_{i=1}^{I} \frac{w^{d}\left(q, \vartheta_{i}^{d}\right)}{\sum_{k=1}^{I} w^{d}\left(q, \vartheta_{k}^{d}\right)} D\left(p, q, \vartheta_{i}^{d}\right)=\sum_{j=1}^{J} \frac{w^{s}\left(q, \vartheta_{j}^{s}\right)}{\sum_{k=1}^{J} w^{s}\left(q, \vartheta_{k}^{s}\right)} S\left(p, q, \vartheta_{j}^{s}\right) .
$$

This is easily seen to be equivalent to

$$
\sum_{i=1}^{I} \sum_{j=1}^{J} w^{d}\left(q, \vartheta_{i}^{d}\right) w^{s}\left(q, \vartheta_{j}^{s}\right) D\left(p, q, \vartheta_{i}^{d}\right)=\sum_{i=1}^{I} \sum_{j=1}^{J} w^{d}\left(q, \vartheta_{i}^{d}\right) w^{s}\left(q, \vartheta_{j}^{s}\right) S\left(p, q, \vartheta_{j}^{s}\right),
$$

and using the definition of $F(p, q, \vartheta)$ and $w(q, \vartheta)$ in assumptions $\mathrm{A} 3$ and $\mathrm{A} 4$, we obtain finally that the market clearing equation can be written as:

$$
\sum_{i=1}^{I} \sum_{j=1}^{J} w\left(q, \vartheta_{i}^{d}, \vartheta_{j}^{s}\right) F\left(p, q, \vartheta_{i}^{d}, \vartheta_{j}^{s}\right)=0 .
$$

Introduce $G_{I J}(p, q)$ by

$$
G_{I J}(p, q)=\frac{1}{I J} \sum_{i=1}^{I} \sum_{j=1}^{J} w\left(q, \vartheta_{i}^{d}, \vartheta_{j}^{s}\right) F\left(p, q, \vartheta_{i}^{d}, \vartheta_{j}^{s}\right),
$$

and note that $G_{I J}(p, q)=0$ is equivalent with market equilibrium. The Large Type Limit $G(p, q)$ of $G_{I J}$ is defined by

$$
G(p, q)=\iint w(q, \vartheta) F(p, q, \vartheta) \mathrm{d} \nu(\vartheta),
$$

where $\mathrm{d} \nu(\vartheta)=\mathrm{d} \nu^{d}\left(\vartheta^{d}\right) \mathrm{d} \nu^{s}\left(\vartheta^{s}\right)$. 
Theorem (Existence of evolution law). Under the above assumptions, the equation $G_{I J}(p, q)=$ 0 determines a well defined heterogeneous market evolution law

$$
p=\varphi_{I J}(q)
$$

where $\varphi_{I J}$ depends smoothly on $q$. Likewise, the equation $G(p, q)=0$ determines the Large Type Limit evolution

$$
p=\varphi(q)
$$

where, again, $\varphi$ depends smoothly on $q$.

Proof. This is a simple consequence of the implicit function theorem. Note that since $w(q, \vartheta)>0$, it follows that

$$
\frac{\partial G_{I J}}{\partial p}(p, q)=\frac{1}{I J} \sum_{i=1}^{I} \sum_{j=1}^{J} w\left(q, \vartheta_{i}^{d}, \vartheta_{j}^{s}\right) \frac{\partial F}{\partial p}\left(p, q, \vartheta_{i}^{d}, \vartheta_{j}^{s}\right) \leq \frac{c}{I J} \sum_{i=1}^{I} \sum_{j=1}^{J} w\left(q, \vartheta_{i}^{d}, \vartheta_{j}^{s}\right)<0 .
$$

Hence $G_{I J}$ is strictly monotonically decreasing in $p$, and the equation $G_{I J}(p, q)=0$ can be solved globally for $p$. Note that the smoothness of $\varphi_{I J}$ also follows from the implicit function theorem.

The argument for $G$ is entirely analogous, except for the fact that we have to differentiate under the integral sign. But this is allowed as a consequence of assumption A4 of the previous subsection.

\subsection{Statement of the theorem}

We are now ready to state the main theorem:

Theorem (Convergence almost surely to the LTL) Under assumptions A1-A4, the heterogeneous market evolution law $\varphi_{I J}$ in (5) and its derivatives tend to their Large Type Limit evolution $\varphi$ in (6) and its derivatives almost surely as $I \rightarrow \infty, J \rightarrow \infty$, uniformly on compact sets.

Notice that the large type limit $G(p, q)$ in (4) is simply obtained from the corresponding heterogeneous market function $G_{I J}(p, q)$ in (3), by replacing sample averages by expected values. This suggests application of a uniform law of large numbers. The following theorem is quoted from Jennrich (1969, p. 636, theorem 2). It enables us to replace sums by integrals in the above expressions.

Theorem. Let $g(s, \vartheta)$ be defined on $\mathcal{S} \times \mathcal{E}$, where $\mathcal{E}$ is an Euclidean space, and where $\mathcal{S}$ is a compact subset of an Euclidean space. Let $g$ be continuous in s for each $\vartheta$, and measurable in $\vartheta$ for each $s$. Assume that $|g(s, \vartheta)| \leq h(\vartheta)$ for all $s$ and $\vartheta$, where $h(\vartheta)$ is integrable with respect to a 
probability distribution $\nu$ on $\mathcal{E}$. If $\vartheta_{1}, \vartheta_{2}, \ldots$, is a random sample of realisations of $\Theta$, then for almost every sequence $\left\{\vartheta_{h}\right\}$,

$$
\frac{1}{H} \sum_{h=1}^{H} g\left(\vartheta_{h}, s\right) \rightarrow \int g(\vartheta, s) \mathrm{d} \nu(\vartheta) \quad \text { almost surely, }
$$

uniformly in s.

Proof of the LTL-convergence theorem. This is now a double application of the uniform law of large numbers. First, convergence of $G_{I J}$ in (3) to $G$ in (4) is considered.

Choose $\delta>0, \varepsilon>0$ arbitrarily, and let $K$ be a fixed compact set in $(p, q)$-space. Then, by the uniform law of large numbers, there exists $I_{0}>0$ such that

$$
\begin{aligned}
P\left(\sup _{(p, q) \in K} \mid \frac{1}{I_{0}} \sum_{i=1}^{I_{0}} \int w\left(q, \vartheta_{i}^{d}, \vartheta^{s}\right) F\left(p, q, \vartheta_{i}^{d}, \vartheta^{s}\right) \mathrm{d} \nu^{s}\left(\vartheta^{s}\right)\right. \\
\left.-\iint w(q, \vartheta) F(p, q, \vartheta) \mathrm{d} \nu(\vartheta) \mid \geq \frac{\varepsilon}{2}\right)<\frac{\delta}{2} .
\end{aligned}
$$

Applying the uniform law of large numbers again, we find a $J_{0}>0$, such that

$$
\begin{aligned}
P\left(\sup _{(p, q) \in K} \mid \frac{1}{I_{0}}\right. & \frac{1}{J_{0}} \sum_{i=1}^{I_{0}} \sum_{j=1}^{J_{0}} w\left(q, \vartheta_{i}^{d}, \vartheta_{j}^{s}\right) F\left(p, q, \vartheta_{i}^{d}, \vartheta_{j}^{s}\right) \\
& \left.-\frac{1}{I_{0}} \sum_{i=1}^{I_{0}} \int w\left(q, \vartheta_{i}^{d}, \vartheta^{s}\right) F\left(p, q, \vartheta_{i}^{d}, \vartheta^{s}\right) \mathrm{d} \nu^{s}\left(\vartheta^{s}\right) \mid \geq \frac{\varepsilon}{2}\right)<\frac{\delta}{2}
\end{aligned}
$$

Combining these, it follows that with probability larger than $1-\delta$ the following inequality holds:

$$
\sup _{(p, q) \in K}\left|\frac{1}{I_{0}} \frac{1}{J_{0}} \sum_{i=1}^{I_{0}} \sum_{j=1}^{J_{0}} w\left(q, \vartheta_{i}^{d}, \vartheta_{j}^{s}\right) F\left(p, q, \vartheta_{i}^{d}, \vartheta_{j}^{s}\right)-\iint w(q, \vartheta) F(p, q, \vartheta) \mathrm{d} \nu(\vartheta)\right|<\varepsilon .
$$

This shows that $G_{I J} \rightarrow G$ almost surely as $I, J \rightarrow \infty$, uniformly on compact sets. Note that the same argument shows that $D_{\alpha} G_{I J} \rightarrow D_{\alpha} G$ almost surely, uniformly on compact sets. This in turn implies the almost sure convergence of the heterogeneous market evolution law $\varphi_{I J}$ in (5) and its derivatives to the LTL-evolution law $\varphi$ in (6) and its derivatives, uniformly on compact sets. This completes the proof.

\section{Dynamical consequences}

In what sense is the large type limit evolution a 'good' approximation of the dynamical behaviour of a heterogeneous market with finitely many, but a large number of trader types? This question leads 
to two key concepts in the theory of dynamical systems, structural stability and persistence. These are discussed in a general setting in subsection 3.1, and applied to our heterogeneous market system in subsection 3.2. With probability arbitrarily close to 1 , all generic and persistent properties of the LTL dynamical system also occur for the heterogeneous agent dynamical system if the number of buyer and seller types is sufficiently large.

\subsection{Structural stability and persistence}

In this subsection $\Psi$ and $\Phi$ denote general dynamical systems. A dynamical system $\Psi$ is called $C^{k}$-structurally stable, if every system $\Phi$ sufficiently close to $\Psi$ in the $C^{k}$-norm $\|.\|_{k}$ is conjugated to $\Psi$; that is, by a suitable change of variables and parameters, $\Phi$ can be transformed into $\Psi$.

Structural stability of a system is usually hard to prove. However, if the concept is restricted to certain properties of the system $\Psi$ - like having an attracting fixed point, or undergoing a saddle-node bifurcation - sometimes it can be shown that all systems $\Phi$ sufficiently $C^{k}$-close to $\Psi$ also have the same property, which is then called a $C^{k}$-persistent property. Usually the degree of differentiability $k$ is not explicitly mentioned: 'persistent' means $C^{k}$-persistent with $k$ large enough for the purpose at hand. See e.g. Guckenheimer and Holmes (1986) for a general discussion of structural stability and persistence.

\section{Examples of persistent properties}

The simplest example of a persistent property is a hyperbolic fixed point. That is, if $\Psi$ has a fixed point $x_{0}$, and all eigenvalues of $D_{x} \Psi\left(x_{0}\right)$ are off the unit circle in the complex plane, then $x_{0}$ is called a hyperbolic fixed point. Every $\Phi$ sufficiently close to $\Psi$ in the $\|.\|_{1}$-norm also has a hyperbolic fixed point: hence hyperbolic fixed points are $C^{1}$-persistent.

An important class of examples is furnished by so-called generic bifurcations, like the saddle-node, Hopf, and period-doubling bifurcations of fixed points. Every generic bifurcation has a positive integer $\ell$ associated to it, the co-dimension of the bifurcation; see e.g. Kuznetsov (1998) for an introduction to bifurcation theory and a detailed mathematical treatment of bifurcations of co-dimension 1 and 2. If $\Psi$ depends on an $M$-dimensional parameter $\lambda$ and $\Psi$ has for $\lambda=\lambda_{0}$ a generic codimension- $\ell$ bifurcation with $\ell \leq M$, then any $M$-parameter system $\Phi$ sufficiently close to $\Psi$ in the $C^{k}$-norm (the required degree $k$ is determined by the specific bifurcation) has the same bifurcation, possibly for a different value of the parameter $\lambda=\lambda_{1}$. In short: for a dynamical system depending on $M$ parameters, all generic bifurcations with co-dimension up to and including $M$ are persistent. 
In particular, generic saddle-node, Hopf, and period doubling bifurcations are persistent for 1parameter families of dynamical systems. In 2-parameter systems, cusp and Bogdanov-Takens bifurcations (to name a few) persist.

These are all examples of persistent local properties. An important example of a persistent global property is a so-called transversal homoclinic point, that is, a transversal intersection $p$ of stable and unstable manifolds of a (saddle) fixed point. Homoclinic points are characterized by the fact that its iterates $\varphi^{n}(p)$ approach the fixed point for $n \rightarrow \infty$ as well as for $n \rightarrow-\infty$. Transversal homoclinic points are persistent under small perturbations. Note that the existence of a transversal homoclinic point implies the occurrence of horseshoe dynamics in the system.

The genesis of transversal homoclinic intersections by a homoclinic tangency bifurcation in oneparameter families is another global example of a persistent property. In systems with twodimensional phase space, such a bifurcation implies (under some mild conditions) the existence of strange attractors for a set of parameters of positive measure. See Palis and Takens (1993) for a recent mathematical treatment of homoclinic bifurcation theory. De Vilder (1996) contains a stimulating introduction of phenomena associated to homoclinic bifurcations and application to a two-dimensional version of the overlapping generations model.

Another example is furnished by the Newhouse-Ruelle-Takens phenomenon: they showed that for a family of mappings, having an invariant quasi-periodic circle bifurcating to an invariant 2-torus (a so-called quasi-periodic Hopf bifurcation), by a $C^{2}$-small perturbation, a system with a strange attractor instead of a quasi-periodic 2-torus can be obtained (Ruelle and Takens (1971) and Newhouse, Ruelle and Takens (1978)). Hence every system displaying a quasi-periodic Hopf bifurcation is $C^{2}$-near to a system with a strange attractor. Note that quasi-periodic Hopf bifurcations occur generically in two-parameter families (though only on parameter sets of positive measure, but empty interior, see Broer et al. (1990)).

\section{Examples of non-persistent properties}

A non-persisting example is furnished by the pitchfork bifurcation. It will be useful to discuss this example here, since it will play some role in the simple LTL studied in section 4. A simple example exhibiting a pitchfork bifurcation is the 1-parameter family of 1-dimensional dynamical systems $x_{t}=\Psi\left(x_{t-1}, \lambda\right)$ for $\lambda=0$, where $\Psi$ is given by:

$$
\Psi(x, \lambda)=x+\lambda x-x^{3}, \quad|x|<\frac{1}{3},|\lambda|<\frac{2}{3} .
$$

For $\lambda>0$ there are two stable fixed points $x= \pm \sqrt{\lambda}$ and one unstable fixed point $x=0$, which coalesce at $\lambda=0$. For $\lambda<0$ there is only one stable fixed point $x=0$. 
[Figure 1 about here.]

By adding a small constant $\varepsilon>0$ to $\Psi$, the picture disintegrates. The system $\Psi_{\varepsilon}$, with:

$$
\Psi_{\varepsilon}(x, \lambda)=x+\lambda x-x^{3}+\varepsilon,
$$

has a generic saddle node bifurcation of a stable and an unstable fixed point, together with a stable (hyperbolic) fixed point which exists for all $\lambda$. Hence the pitchfork bifurcation is not generic. In fact, the pitchfork bifurcation only occurs generically in systems with a reflectional symmetry. For example, the system (7) is symmetric w.r.t. to $x=0$, since $\Psi(-x, \lambda)=-\Psi(x, \lambda)$. Adding a perturbation parameter $\varepsilon$ in (8) means a breaking of the symmetry in the system and the pitchfork bifurcation disappears. It should be noted however that for small $\varepsilon$ the bifurcation diagram of the perturbed pitchfork in figure 2 is close to the bifurcation diagram of the pitchfork in figure 1

\subsection{Application to large type limits}

The above considerations can be applied to heterogeneous market systems with many trader types and their corresponding large type limit (LTL).

\section{Heterogeneous market and LTL dynamical systems}

First we have to be more precise about the dynamical systems corresponding to the heterogeneous market with $I$ buyers and $J$ sellers and the dynamical system corresponding to its LTL. Recall that the heterogeneous market evolution map determining the market equilibrium price $p=p_{t}$ at date $t$ is of the form

$$
p=\varphi_{I J}\left(x, \lambda ; \vartheta_{1}^{d}, \ldots \vartheta_{I}^{d}, \vartheta_{1}^{s}, \ldots \vartheta_{J}^{s}\right)
$$

where we have extended the notation as follows. We split the vector $q$ into $x$ and $\lambda$, with $x$ denoting the vector of past state variables (in applications typically a vector $\left(p_{t-1}, \cdots, p_{t-L}\right)$ of past prices, or their deviations from a fundamental price $p^{*}$ ) and $\lambda$ denoting a vector of structural parameters. Moreover, we included the parameters $\vartheta_{i}^{d}, \vartheta_{i}^{s}$ of all $I$ individual buyers and $J$ individual sellers, which were drawn independently from the probability distributions $\nu^{d}$ and $\nu^{s}$. In order to write down the corresponding dynamical system for this heterogeneous agents market, we have to be precise about the future states of all state variables contained in the vector $x$. Although this can be done more generally, in order to keep the notation simple we restrict our attention here to the case where $x$ is a finite dimensional vector of past prices only. This will be sufficient for our purposes, and our heterogeneous asset market application in section 4 will be of this form. 
If the state $\mathbf{x}_{t} \equiv\left(x_{1 t}, x_{2 t}, \ldots, x_{L t}\right)$ at time $t$ is known, the state $\mathbf{x}_{t+1}$ at time $t+1$ is given by $\mathbf{x}_{t+1}=$ $\Phi_{I J}\left(\mathbf{x}_{t}, \lambda ; \vartheta\right)$, where

$$
\Phi_{I J}(\mathbf{x}, \lambda ; \vartheta)=\left(\varphi_{I J}(\mathbf{x}, \lambda ; \vartheta), x_{1}, \ldots, x_{L-1}\right)
$$

with $\vartheta=\left(\vartheta_{1}^{d}, \ldots \vartheta_{I}^{d}, \vartheta_{1}^{s}, \ldots \vartheta_{J}^{s}\right)$. The map $\Phi_{I J}$ defines the dynamical system associated to the evolution map $\varphi_{I J}$ describing the dynamics of a heterogeneous market with $I$ buyers and $J$ sellers.

This dynamical system is very complicated, because $\vartheta$ contains $I+J$ individual parameter vectors $\vartheta_{i}^{d}$ and $\vartheta_{j}^{s}$ drawn randomly from probability measures $\nu^{d}$ and $\nu^{s}$. These individual parameter vectors $\vartheta_{i}^{d}$ and $\vartheta_{j}^{s}$ are drawn once, in the beginning of the market, and thereafter compete against each other according to the heterogeneous market dynamics described by (10).

Let us now discuss the LTL dynamical system. Recall that the LTL evolution map $\varphi$ determining the market equilibrium price $p=p_{t}$ at date $t$ is given by

$$
p=\varphi(x, \lambda ; \nu)
$$

where $q$ has been split into a vector of past state variables $x$ and a vector of structural parameters $\lambda$, as before, and where we have also included the probability measure $\nu$, from which all individual parameter vectors $\left(\vartheta_{i}^{d}, \vartheta_{j}^{s}\right)$ have been drawn, in the notation. In applications, one can work with parameterized distribution functions $\nu=\nu_{\alpha}$, such as a multivariate normal distribution, and the LTL evolution map $\varphi(x, \lambda ; \alpha)$ will then depend upon the parameter vector $\alpha$. The LTL dynamical system reads as

$$
\Phi(\mathbf{x}, \lambda ; \alpha)=\left(\varphi(\mathbf{x}, \lambda ; \alpha), x_{1}, \ldots, x_{L-1}\right)
$$

We will refer to $\lambda$ as the structural parameter and to $\alpha$ as the heterogeneity parameter.

\section{Persistence of bifurcations 'in probability'}

According to the LTL-theorem the heterogeneous market evolution map $\varphi_{I J}$, together with its derivatives up to order $k$, converge almost surely to the LTL evolution map $\varphi$ and its derivatives, as $I$ and $J$ tend to infinity. From this it follows immediately that the heterogeneous market dynamics map $\Phi_{I J}(10)$, together with the derivatives up to order $k$, also converge almost surely to the LTL dynamics map $\Phi(12)$ and its derivatives, as $I$ and $J$ tend to infinity.

Let $\varepsilon \in(0,1)$ and let the heterogeneity parameter $\alpha$ be fixed. Since generic co-dimension- $\ell$ bifurcations persist as long as $\ell$ does not exceed the number of structural parameters, it follows that any generic co-dimension $\ell$ bifurcation with respect to the structural parameter $\lambda$ for $\Psi$ ( $\sigma$ is fixed) occurs with probability at least $1-\varepsilon$ for $\Phi_{I J}$, for $I$ and $J$ large. 


\section{The role of heterogeneity parameters}

Note the distinction between structural parameters $\lambda$ and heterogeneity parameters $\alpha$. This is roughly due to the fact that for $\Phi_{I J}$ and $\Psi$, only bifurcations in $\lambda$ can be compared with each other, since $\Phi_{I J}$ does not depend in a direct way on $\alpha$. The following example may illustrate this point further. Suppose $\Phi_{I J}(x ; \vartheta)$ denotes the following dynamical system:

$$
\Phi_{H}(x ; \vartheta)=\frac{1}{H} \sum_{h=1}^{H} \vartheta_{h}+x-x^{2},
$$

with $\vartheta_{h}$ IID, $\mathrm{E}_{\nu} \vartheta_{h}=\mu$ and $\operatorname{Var} \vartheta_{h}=\sigma^{2}$. The parameters $\alpha=(\mu, \sigma)$ are heterogeneity parameters.

The large type limit $\Psi$ of $\Phi_{I J}$ is given by:

$$
\Psi(x ; \alpha)=\mu+x-x^{2} .
$$

For $\mu>0$, this system has two hyperbolic fixed points; for $\mu<0$, there are none. At $\mu=0$, there is one non-hyperbolic fixed point. Hence, the large type limit exhibits a saddle-node bifurcation of steady states at the heterogeneity parameter $\mu=0$.

Now, let again $\varepsilon \in(0,1)$ and $\mu>0$ be fixed. Set $\delta=\frac{\mu}{2}$. Then from the LTL convergence theorem and the discussion above it follows that there are $I_{0}, J_{o}>0$, such that for all $I \geq I_{0}$ and $J \geq J_{0}$ :

$$
\left\|\Psi-\Phi_{I J}\right\|_{k}=\left|\sum_{h=1}^{H} \vartheta_{h}-\mu\right| \leq \delta,
$$

with probability at least $1-\varepsilon$. (Of course, the same result can be found directly by Chebyshev's inequality). Hence, with this probability:

$$
\sum_{h=1}^{H} \vartheta_{h} \geq \frac{\mu}{2}>0,
$$

and the system $\Phi_{I J}(x ; \vartheta)$ has two fixed points.

On the other hand, if $\mu$ is set to 0 , then asymptotically:

$$
\frac{1}{H} \sum \vartheta_{h} \sim N\left(0, \sigma^{2} / H\right)
$$

where $N\left(0, \sigma^{2} / H\right)$ denotes the normal distribution around 0 . Hence the probabilities of $\frac{1}{H} \sum \vartheta_{h}>$ 0 and $\frac{1}{H} \sum \vartheta_{h}<0$ are both approximately $\frac{1}{2}$. This simple example shows that, at the saddle-node bifurcation value of the heterogeneity parameter $\mu=0$, the large type limit furnishes no information about the number of steady states of the finite belief systems.

We summarize this as follows: bifurcations in the large type limit system may be found using heterogeneity parameters, which may yield useful information e.g. concerning number and/or stability 
of steady states. However, only bifurcations of the LTL w.r.t. structural parameters, with the heterogeneity parameters fixed at non-bifurcation values, can be directly compared to corresponding bifurcations in the heterogeneous market with many trader types.

\section{Corollary of large type limit convergence theorem}

The discussion in the present section is summarized in the following corollary, listing some important persistent properties which, if they occur for the LTL, with high probability also occur for the heterogeneous market system when $I$ and $J$ are large.

\section{Corollary of LTL convergence theorem}

Let $\Phi_{I J}$ in (10) be the dynamical system with I buyer and J seller types and $M$ structural parameters, and let $\Psi$ in (12) be its Large Type Limit as $I$ and $J$ tend to infinity. Then for every $k$ and every $C^{k}$ persistent property of $\Psi$ and for every $\varepsilon$, there exist numbers $I_{0}, J_{0}$ of buyer and seller types, such that for every $I \geq I_{0}$ and $J \geq J_{0}$, with probability $\geq 1-\varepsilon$ the dynamical system $\Phi_{I J}$ generically has the same property.

In particular, this means that if $\Psi$ has

1. hyperbolic fixed or hyperbolic periodic points,

2. transversal homoclinic intersections of stable and unstable manifolds of a fixed or periodic (saddle) point,

3. horseshoes,

4. local or global bifurcations up to co-dimension $M$,

5. open neighborhoods in function space with strange attractors (Newhouse-Ruelle-Takens phenomenon),

then $\Phi_{I J}$ will have these too with probability $\geq 1-\varepsilon$ if $I \geq I_{0}$ and $J \geq J_{0}$.

\section{A financial market with heterogeneous beliefs}

This section presents a simple application of the notion of LTL, namely a standard asset pricing model with heterogeneous beliefs as introduced in Brock and Hommes (1998). Subsection 4.1 recalls the asset pricing model, whereas in subsection 4.2 we derive its LTL and study the dynamical behaviour of the LTL. 


\subsection{The asset pricing model with heterogeneous beliefs}

This subsection briefly recalls the notion of Adaptive Belief System (ABS), as introduced by Brock and Hommes (1998), applying the evolutionary framework developed in Brock and Hommes (1997a) to a standard asset pricing model; see also Brock (1997) and Hommes (2001) for more extensive discussions. An ABS is in fact a standard discounted value asset pricing model derived from meanvariance maximization, extended to the case of heterogeneous beliefs. Agents can either invest in a risk free asset or in a risky asset. The risk free asset is perfectly elastically supplied and pays a fixed rate of return $r$; the risky asset, for example a large stock or a market index, pays an uncertain dividend. Let $p_{t}$ be the price per share (ex-dividend) of the risky asset at time $t$, and let $y_{t}$ be the stochastic dividend process of the risky asset. Wealth dynamics is given by

$$
\tilde{W}_{t+1}=R W_{t}+\left(\tilde{p}_{t+1}+\tilde{y}_{t+1}-R p_{t}\right) z_{t},
$$

where $R=1+r$ is the gross rate of risk free return, variables with a tilde such as $\tilde{y}_{t+1}$ denote random variables at date $t+1$ and $z_{t}$ denotes the number of shares of the risky asset purchased at date $t$. Let $E_{t}$ and $V_{t}$ denote the conditional expectation and conditional variance based on a publically available information set such as past prices and past dividends. Let $E_{h t}$ and $V_{h t}$ denote the 'beliefs' or forecasts of trader type $h$ about conditional expectation and conditional variance. Agents are assumed to be myopic mean-variance maximizers so that the demand $z_{h t}$ of type $h$ for the risky asset solves

$$
\operatorname{Max}_{z_{t}}\left\{E_{h t}\left[\tilde{W}_{t+1}\right]-\frac{a}{2} V_{h t}\left[\tilde{W}_{t+1}\right]\right\}
$$

where $a$ is the risk aversion parameter. The demand $z_{h t}$ for risky assets by trader type $h$ is then

$$
z_{h t}=\frac{E_{h t}\left[\tilde{p}_{t+1}+\tilde{y}_{t+1}-R p_{t}\right]}{a V_{h t}\left[\tilde{p}_{t+1}+\tilde{y}_{t+1}-R p_{t}\right]}=\frac{E_{h t}\left[\tilde{p}_{t+1}+\tilde{y}_{t+1}-R p_{t}\right]}{a \sigma^{2}},
$$

where the conditional variance $V_{h t}=\sigma^{2}$ is assumed to be equal for all types and constant. ${ }^{2}$ Let $z^{s}$ denote the supply of outside risky shares per investor, assumed to be constant, and let $n_{h t}$ denote the fraction of type $h$ at date $t$. Equilibrium of demand and supply yields

$$
\sum_{h=1}^{H} n_{h t} \frac{E_{h t}\left[\tilde{p}_{t+1}+\tilde{y}_{t+1}-R p_{t}\right]}{a \sigma^{2}}=z^{s},
$$

where $H$ is the number of different trader types. The forecasts $E_{h t}\left[\tilde{p}_{t+1}+\tilde{y}_{t+1}\right]$ of tomorrows prices and dividends are made before the equilibrium price $p_{t}$ has been revealed by the market and therefore will depend upon a publically available information set $I_{t-1}=\left\{p_{t-1}, p_{t-2}, \ldots ; y_{t-1}, y_{t-2}, \ldots\right\}$ of

\footnotetext{
${ }^{2}$ Gaunersdorfer (2000) investigates the case with time varying beliefs about variances and shows that the results are quite similar to those for constant variance.
} 
past prices and dividends. Market equilibrium (16) then implies that the realized market price $p_{t}$ will be the unique price for which demand equals supply.

Notice that in the heterogeneous market equilibrium equation (16) the outside supply of shares is constant, and in this application heterogeneity thus only appears on the demand side, with $H$ different types of traders. Solving the heterogeneous market equilibrium equation for the equilibrium price gives

$$
R p_{t}=\sum_{h=1}^{H} n_{h t} E_{h t}\left[\tilde{p}_{t+1}+\tilde{y}_{t+1}\right]-a \sigma^{2} z^{s} .
$$

The quantity $a \sigma^{2} z^{s}$ may be interpreted as a risk premium for traders to hold all risky assets.

\section{The EMH benchmark with rational agents}

Let us first discuss the EMH-benchmark with rational expectations. In a world where all traders are identical and expectations are homogeneous the arbitrage market equilibrium equation (17) reduces to

$$
R p_{t}=E_{t}\left[\tilde{p}_{t+1}+\tilde{y}_{t+1}\right]-a \sigma^{2} z^{s}
$$

where $E_{t}$ denotes the common conditional expectation of all traders at the beginning of period $t$, based on a publically available information set $I_{t}$. It is well known that, using the market equilibrium equation (18) repeatedly and assuming that the transversality condition

$$
\lim _{t \rightarrow \infty} \frac{E_{t}\left[\tilde{p}_{t+k}\right]}{R^{k}}=0
$$

holds, the price of the risky asset is uniquely determined by

$$
p_{t}^{*}=\sum_{k=1}^{\infty} \frac{E_{t}\left[\tilde{y}_{t+k}\right]-a \sigma^{2} z^{s}}{R^{k}} .
$$

The price $p_{t}^{*}$ in (20) is called the EMH fundamental rational expectations (RE) price, or the fundamental price for short. The fundamental price is completely determined by economic fundamentals and given by the discounted sum of expected future dividends minus the risk premium. In general, the properties of the fundamental price $p_{t}^{*}$ depend upon the stochastic dividend process $y_{t}$. We will mainly focus on the case of an IID dividend process $y_{t}$, with constant mean $E\left[y_{t}\right]=\bar{y}$. We note however that any other random dividend process $y_{t}$ may be substituted in what follows ${ }^{3}$. For an IID dividend process $y_{t}$ with constant mean, the fundamental price is constant and given by

$$
p^{*}=\sum_{k=1}^{\infty} \frac{\bar{y}-a \sigma^{2} z^{s}}{R^{k}}=\frac{\bar{y}-a \sigma^{2} z^{s}}{r} .
$$

\footnotetext{
${ }^{3}$ Brock and Hommes (1997b) for example discuss a non-stationary example, where the dividend process is a geometric random walk .
} 


\section{Heterogeneous beliefs}

It will be convenient to work with the deviation from the fundamental price, that is, with

$$
x_{t}=p_{t}-p_{t}^{*} \text {. }
$$

We make the following assumptions about the beliefs of trader type $h$ :

B1 $V_{h t}\left[\tilde{p}_{t+1}+\tilde{y}_{t+1}-R p_{t}\right]=V_{t}\left[\tilde{p}_{t+1}+\tilde{y}_{t+1}-R p_{t}\right]=\sigma^{2}$, for all $h, t$.

B2 $E_{h t}\left[\tilde{y}_{t+1}\right]=E_{t}\left[\tilde{y}_{t+1}\right]=\bar{y}$, for all $h, t$.

B3 All beliefs $E_{h t}\left[\tilde{p}_{t+1}\right]$ are of the form

$$
E_{h t}\left[\tilde{p}_{t+1}\right]=E_{t}\left[\tilde{p}_{t+1}^{*}\right]+E_{h t}\left[\tilde{x}_{t+1}\right]=p^{*}+f_{h}\left(x_{t-1}, \ldots, x_{t-L}\right), \quad \text { for all } h, t .
$$

According to assumption B1 beliefs about conditional variance are equal and constant for all types, as discussed above already. Assumption B2 states that expectations about future dividends $\tilde{y}_{t+1}$ are the same for all trader types and equal to the conditional expectation, which is $\bar{y}$ in the case of IID dividends. According to assumption B3, beliefs about future prices consist of two parts: a common belief about the fundamental plus a heterogeneous part for each type $h$. According to assumption B3, all traders know the fundamental price $p^{*}$ but traders nevertheless believe that in a heterogeneous world prices may deviate from their fundamental value $p^{*}$ by some function $f_{h}$ depending upon past deviations from the fundamental. A frequently heard (theoretical) argument is why would some traders behave differently, given the same information? One may as well turn this argument on its head: why would all traders behave the same, given the same information? It is a well known fact in behavioural economics by now that economic agents may decide differently even when given the same information. In our framework, given the same information about economic fundamentals, we allow for differences in beliefs about future asset prices. Realized market prices and beliefs will co-evolve over time and evolution will decide who is right, as discussed below.

Each forecasting rule $f_{h}$ in (23) represents the model of the market according to which type $h$ believes that prices will deviate from the commonly shared fundamental price. For example, a forecasting strategy $f_{h}$ may correspond to a technical trading rule, based upon short run or long run moving averages, of the type used in real markets. The function $f_{h}$, expressing the belief of type $h$ on the price of the risky asset at time $t+1$, is assumed to have the following general form:

$$
f_{h t}=f\left(x_{t-1}, \ldots, x_{t-d}, \lambda, \vartheta_{h}\right)
$$

The $x_{t-j}, j=1, \ldots, d$, are the past price deviations up to the maximal time delay $d$, as above $\lambda$ is a multidimensional structural parameter (containing all economic parameters, such as the interest rate 
$r$ or the mean dividend $\bar{y}$, that may be used in the forecasting functions), and the belief variable $\vartheta_{h}$ is a multidimensional stochastic variable which characterizes the belief $h$. In this application, heterogeneity is thus parameterized by the stochastic belief variable $\vartheta_{h}$. At the beginning of the market, the beliefs $\vartheta_{h}$ are sampled from a general distribution of beliefs; thereafter these beliefs compete against each other according to some evolutionary dynamics to be discussed below.

An important and convenient consequence of the assumptions B1-B3 concerning traders' beliefs is that the heterogeneous agent market equilibrium equation (17) can be reformulated in deviations from the benchmark fundamental. In particular substituting the price forecast (23) in the market equilibrium equation (17) and using the fact that the fundamental price $p_{t}^{*}$ satisfies $R p_{t}^{*}=E_{t}\left[p_{t+1}^{*}+\right.$ $\left.y_{t+1}\right]-a \sigma^{2} z^{s}$ yields the equilibrium equation in deviations from the fundamental:

$$
R x_{t}=\sum_{h=1}^{H} n_{h t} E_{h t}\left[\tilde{x}_{t+1}\right] \equiv \sum_{h=1}^{H} n_{h t} f_{h t},
$$

with $f_{h t}=f_{h}\left(x_{t-1}, \ldots, x_{t-L}\right)$. An important reason for our model formulation in terms of deviations from a benchmark fundamental is that in this general setup, it is immediately apparant how the benchmark rational expectations asset pricing model is nested in our model as the special case having all forecasting strategies $f_{h} \equiv 0$. In this way, the adaptive belief systems can be used easily in empirical and experimental testing of whether asset prices deviate significantly from anyone's favorite benchmark fundamental.

\section{Evolutionary dynamics}

The evolutionary part of the model describes how beliefs are updated over time, that is, how the fractions $n_{h t}$ of trader types in the market equilibrium equation (25) evolve over time. Fractions are updated according to an evolutionary fitness or performance measure. The fitness measures of all trading strategies are publically available, but subject to noise. Fitness is derived from a random utility model and given by

$$
\tilde{U}_{h t}=U_{h t}+\epsilon_{h t}
$$

where $U_{h t}$ is the deterministic part of the fitness measure and $\epsilon_{h t}$ represents IID noise across $h=$ $1, \ldots H$. In order to obtain analytical expressions for the probabilities or fractions, it will be assumed that the noise $\epsilon_{h t}$ is drawn from a double exponential distribution. In that case, in the limit as the number of agents goes to infinity, the probability that an agent chooses strategy $h$ is given by the 
well known discrete choice model or 'Gibbs' probabilities ${ }^{4}$

$$
n_{h t}=\frac{e^{\beta U_{h, t-1}}}{\sum_{h=1}^{H} e^{\beta U_{h, t-1}}} .
$$

Note that the fractions $n_{h t}$ add up to 1 . The crucial feature of (27) is that the higher the fitness of trading strategy $h$, the more traders will select strategy $h$. The parameter $\beta$ in (27) is called the intensity of choice; it controls the sensitivity of the mass of traders to selecting the optimal prediction strategy. The intensity of choice $\beta$ is inversely related to the variance of the noise terms $\epsilon_{h t}$. The extreme case $\beta=0$ corresponds to the case of infinite variance noise, so that differences in fitness cannot be observed and all fractions (27) will be fixed over time and equal to $1 / H$. The other extreme case $\beta=+\infty$ corresponds to the case without noise, so that the deterministic part of the fitness can be observed perfectly and in each period, all traders choose the optimal forecast. An increase in the intensity of choice $\beta$ represents an increase in the degree of rationality with respect to evolutionary selection of trading strategies. The timing of the coupling between the market equilibrium equation (17) or (25) and the evolutionary selection of strategies (27) is crucial. The market equilibrium price $p_{t}$ in (17) depends upon the fractions $n_{h t}$. The notation in (27) stresses the fact that these fractions $n_{h t}$ depend upon most recently observed past fitnesses $U_{h, t-1}$, which in turn depend upon past prices $p_{t-1}$ and dividends $y_{t-1}$ in periods $t-1$ and further in the past, as will be seen below. After the equilibrium price $p_{t}$ has been revealed by the market, it will be used in evolutionary updating of beliefs and determining the new fractions $n_{h, t+1}$. These new fractions will then determine a new equilibrium price $p_{t+1}$, etc. In the Adaptive Belief System, market equilibrium prices and fractions of different trading strategies thus co-evolve over time.

A natural candidate for evolutionary fitness is accumulated realized profits, as given by 5

$$
U_{h t}=\left(\tilde{p}_{t}+\tilde{y}_{t}-R p_{t-1}\right) \frac{E_{h, t-1}\left[\tilde{p}_{t}+\tilde{y}_{t}-R p_{t-1}\right]}{a \sigma^{2}}+w U_{h, t-1},
$$

where $R=1+r$ is the gross risk free rate of return, and $0 \leq w \leq 1$ is a memory parameter measuring how fast past realized fitness is discounted for strategy selection. The first term in (28)

\footnotetext{
${ }^{4}$ See Manski and McFadden (1981) and Anderson, de Palma and Thisse (1993) for extensive discussion of discrete choice models and their applications in economics.

${ }^{5}$ Given that investors are risk averse mean-variance maximizers, maximizing their expected utility from wealth another natural candidate for fitness are the risk adjusted profits. In fact, the fitness measure (28) based upon realized profits does not take into account the variance term in (14) capturing the investors' risk taken before obtaining that profit. On the other hand, in real markets realized net profits or accumulated wealth may be what investors care about most, and the non-risk adjusted fitness measure (28) may thus be practically important. See BH 1998 and Hommes (2001) for a discussion of this point. Gaunersdorfer, Hommes and Wagener (2000) investigate an evolutionary model with fitness given by risk adjusted profits. In any event the methodology of LTL developed in this paper can be developed for alternative fitness functions to (28). The general point that taking the LTL drastically reduces the number of "parameters" in heterogeneous belief models with large numbers of such beliefs to the small set of parameters that determine "underlying characteristics" of underlying "belief distributions" remains independently of what choice is made for the fitness function.
} 
represents the profit that type $h$ realised in the last period, which is given by the realized excess return of the risky asset over the risk free asset, times the demand for the risky asset by traders of type $h$. In the extreme case with no memory, that is with $w=0$, fitness $U_{h t}$ equals net realized profit in the previous period, whereas in the other extreme case with infinite memory, that is with $w=1$, fitness $U_{h t}$ equals total wealth as given by accumulated realized profits over the entire past. In the intermediate case, the weight given to past realized profits decreases exponentially with time.

Fitness can now be rewritten in terms of deviations from the fundamental as

$$
U_{h t}=\left(\tilde{x}_{t}-R x_{t-1}+a \sigma^{2} z^{s}+\delta_{t}\right)\left(\frac{f_{h, t-1}-R x_{t-1}+a \sigma^{2} z^{s}}{a \sigma^{2}}\right)+w U_{h, t-1},
$$

where $\delta_{t} \equiv \tilde{p}_{t}^{*}+\tilde{y}_{t}-E_{t-1}\left[\tilde{p}_{t}^{*}+\tilde{y}_{t}\right]$ is a martingale difference sequence.

\subsection{The Large Type Limit of the asset pricing model}

In this subsection we derive the Large Type Limit (LTL) of the asset pricing model with heterogeneous beliefs. Recall that the heterogeneous asset market equilibrium equation is given by

$$
\sum_{h=1}^{H} n_{h t} \frac{E_{h t}\left[\tilde{p}_{t+1}+\tilde{y}_{t+1}-R p_{t}\right]}{a \sigma^{2}}=z^{s} .
$$

This is a special case of the general heterogeneous market equilibrium equation (1). In the heterogeneous asset market, the supply of outside shares is fixed and heterogeneity only appears on the demand side. The reader may check that assumptions A1-A3 are satisfied: heterogeneity is parametrized by the stochastic vector $\vartheta_{h}$ of the forecasting rule or belief of type $h$ (A1); the discrete choice fractions $n_{h t}$ satisfy the general assumptions on the weight of each type (A2); the demand function $E_{h t}\left[\tilde{p}_{t+1}+\tilde{y}_{t+1}-R p_{t}\right] /\left(a \sigma^{2}\right)$ of type $h$ is a decreasing function of the market price $p_{t}$, so that a unique market equilibrium price $p_{t}$ always exists, as derived in (17) (A3). Furthermore, we shall assume assumption (A4) to hold, which demands that all relevant functions are smooth and Lebesgue dominated by a positive random variable with finite expected value. In the examples below, typically all systems are smooth and all random variables normal, so that the condition is easily seen to hold.

Consequently, the LTL can be derived using the general framework in section 2. It will be instructive however to see how the LTL can be derived directly from the heterogeneous asset market equilibrium equation. The starting point is the expression for the market equilibrium price (17), rewritten in deviations from the fundamental in (25):

$$
R x_{t}=\sum_{h=1}^{H} n_{h t} f_{h t} .
$$


As before, $x_{t}$ is the deviation from the fundamental price, $n_{h t}$ are the fractions of type $h$ traders and $f_{h t}$ is the function representing the belief of type $h$ on the price of the risky asset at time $t+1$, assumed to have the general form

$$
f_{h t}=f\left(x_{t-1}, \ldots, x_{t-d}, \lambda, \vartheta_{h}\right)
$$

where the belief variable $\vartheta_{h}$ is a multidimensional stochastic variable which characterizes the belief $h$. This belief is sampled from a general distribution of beliefs.

Recall that the fractions $n_{h t}$ are given by the discrete choice model probabilities

$$
n_{h t}=\frac{e^{\beta U_{h, t-1}}}{\sum_{h=1}^{H} e^{\beta U_{h, t-1}}} .
$$

Since the fitness function $U_{h, t-1}$ of type $h$ depends upon $x_{t-1}, x_{t-2}$ and upon the forecasting function $f_{h, t-2}$, their general form is:

$$
U_{h, t-1}=U\left(x_{t-1}, x_{t-2}, \ldots, x_{t-d-2}, \lambda, \vartheta_{h}\right)
$$

where as before $\lambda$ is a structural parameter.

The equilibrium equation (31) can be rewritten to:

$$
x_{t}=\frac{1}{R} \frac{\sum_{h=1}^{H} e^{\beta U_{h, t-1}} f_{h t}}{\sum_{h=1}^{H} e^{\beta U_{h, t-1}}} .
$$

The equilibrium equation (33) determines the evolution of the system with $H$ trader types - this information is coded in the evolution $\operatorname{map} \varphi_{H}(\mathbf{x}, \lambda, \vartheta):^{6}$

$$
\varphi_{H}(\mathbf{x}, \lambda, \vartheta)=\frac{1}{R} \frac{\sum_{h=1}^{H} e^{\beta U\left(\mathbf{x}, \lambda, \vartheta_{h}\right)} f\left(\mathbf{x}, \lambda, \vartheta_{h}\right)}{\sum_{h=1}^{H} e^{\beta U\left(\mathbf{x}, \lambda, \vartheta_{h}\right)}} .
$$

The structural parameter $\lambda=\left(\beta, R, \lambda_{3}, \ldots, \lambda_{q}\right)$ takes values in a bounded open subset $\Lambda$ of $\mathbf{R}^{q}$, and contains all structural parameters of the evolutionary, heterogeneous agents economy, such as the intensity of choice $\beta$, the interest rate $r$ (or gross rate of return $R=1+r$ ), the risk aversion coefficient $a$, etc.

The evolution map gives rise to a dynamical system in the following way. The state variables $\mathbf{x}_{t}=$ $\left(x_{1 t}, \ldots, x_{d+2, t}\right)$ are introduced by setting $x_{j t}=x_{t-j}, 1 \leq j \leq d+2$, where $d$ is the (maximum) number of lags in the forecasting function. The state space $X$ is taken to be the open subset of $\mathbf{R}^{d+2}$

\footnotetext{
${ }^{6}$ In the discussion below the evolution map $\varphi_{H}$ plays the role of the general heterogeneous agents evolution map $\varphi_{T} J$ in (9) and the dynamical system $\Phi_{H}$ in (34) with $H$ belief types plays the role of the general dynamical system $\Phi_{I J}$ in (35) with $I$ buyer and $J$ seller types. The corresponding LTL evolution map and the LTL dynamical systems are denoted by $\varphi$ and $\Phi$ as before.
} 
formed by $\left\{x \in \mathbf{R}^{d+2} \mid x_{i}>-p^{*}\right\}$. If the state $\mathbf{x}_{t}$ at time $t$ is known, the state $\mathbf{x}_{t+1}$ at time $t+1$ is given by $\mathbf{x}_{t+1}=\Phi_{H}\left(\mathbf{x}_{t}, \lambda, \vartheta\right)$, where:

$$
\Phi_{H}(\mathbf{x}, \lambda, \vartheta)=\left(\varphi_{H}(\mathbf{x}, \lambda, \vartheta), x_{1}, \ldots, x_{d+1}\right) .
$$

The map $\Phi_{H}$ defines the dynamical system associated to the evolution map $\varphi_{H}$ corresponding to an asset market with $H$ different belief types. When the number of trader types $H$ is large, the dynamical system (35) contains a large number of stochastic variables $\vartheta=\left(\vartheta_{1}, \ldots, \vartheta_{H}\right)$, where the $\vartheta_{j}$ are independently and identically distributed (henceforth: IID), with distribution $\nu_{\alpha}$. The distribution function of the stochastic belief variable $\vartheta_{h}$ depends on a multi-dimensional parameter $\alpha$, called the belief parameter, which takes values in a bounded open subset $M$ of $\mathbf{R}^{r}$. This setup allows to vary the population out of which the individual beliefs are sampled. The explicit dependence of a probability on $\alpha$ is denoted by $P=P_{\alpha}$.

\section{The limit evolution}

Observe that both the denominator and the numerator of the evolution map $\varphi_{H}$ in (34) may be divided by the number of trader types $H$ and thus may be seen as sample means. The evolution map $\psi$ of the large type limit is then simply obtained by replacing the sample means in the evolution map $\varphi_{H}$ by population means, to obtain:

$$
\psi(\mathbf{x}, \lambda, \mu)=\frac{1}{R} \frac{\mathrm{E}_{\mu}\left[e^{\beta U\left(\mathbf{x}, \lambda, \vartheta_{0}\right)} f\left(\mathbf{x}, \lambda, \vartheta_{0}\right)\right]}{\mathrm{E}_{\mu}\left[e^{\beta U\left(\mathbf{x}, \lambda, \vartheta_{0}\right)}\right]}=\frac{\int e^{\beta U\left(\mathbf{x}, \lambda, \vartheta_{0}\right)} f\left(\mathbf{x}, \lambda, \vartheta_{0}\right) d \nu_{\alpha}}{\int e^{\beta U\left(\mathbf{x}, \lambda, \vartheta_{0}\right)} d \nu_{\alpha}} .
$$

Here $\vartheta_{0}$ is a stochastic variable which is distributed in the same way as the $\vartheta_{h}$, with distribution function $\nu_{\alpha}$. The structural parameter vectors $\lambda$ of the evolution map $\varphi_{H}$ and of the LTL evolution map $\psi$ coincide. However, whereas the evolution map $\varphi_{H}$ in (34) of the heterogeneous agent system contains $H$ randomly drawn multi-dimensional stochastic variables $\vartheta_{h}$, the LTL evolution map $\psi$ in (36) only contains the belief parameter vector $\alpha$ describing the joint probability distribution. Taking a large type limit thus leads to a huge reduction in stochastic belief variables. In section 4.3 we will consider an example where the randomly drawn beliefs are linear, with multi-variate normally distributed belief variables, and the LTL contains as belief parameters the means and the variances of the corresponding multi-variate distribution.

The dynamical system corresponding to the LTL evolution map $\psi$ is denoted by $\Psi(\mathbf{x}, \lambda, \alpha)$ and given by:

$$
\Psi(\mathbf{x}, \lambda, \alpha)=\left(\psi(\mathbf{x}, \lambda, \alpha), x_{1}, \ldots, x_{d+1}\right)
$$


According to the LTL theorem, the LTL dynamical system (37) is a good approximation of the dynamical behaviour in a heterogeneous asset market (35) when the number of belief types $H$ is large, in the sense that all 'generic' and 'persistent' dynamic properties will be preserved with high probability.

\subsection{Example}

In this section we investigate the dynamical behavior of a simple but typical example of an LTL, focusing on generic and persistent properties. Traders' forecasting rules are linear functions of past price deviations, that is

$$
f_{h t}=f_{t}\left(\vartheta_{h}\right)=\vartheta_{h 0}+\vartheta_{h 1} x_{t-1}+\cdots+\vartheta_{h d} x_{t-d}
$$

with stochastic belief variables $\vartheta_{j h}$ distributed multivariate normal. We calculate LTL's for the simple and tractable case where fitness is last period's realized profits, that is,

$$
U_{h t}=\left(x_{t}-R x_{t-1}+a \sigma^{2} z^{s}+\delta_{t}\right)\left(\frac{f_{h, t-1}-R x_{t-1}+a \sigma^{2} z^{s}}{a \sigma^{2}}\right) .
$$

Including more memory in the fitness measure is straightforward, but leads to a high dimensional system $^{7}$. Recall that $\left\{\delta_{t}\right\}$ is a martingale difference sequence. On setting $\delta_{t}=0$, fitness simplifies to

$$
U_{h t}=\left(x_{t}-R x_{t-1}+a \sigma^{2} z^{s}\right)\left(\frac{f_{h, t-1}-R x_{t-1}+a \sigma^{2} z^{s}}{a \sigma^{2}}\right),
$$

and we obtain what will be called "the deterministic skeleton" of the stochastic dynamical system. We wish to analyze stabilizing and destabilizing economic forces by studying this skeleton. It corresponds to the case where the fitness measure equals the conditional expectation of profits.

The fractions $n_{h t}$ of trader type $h$, as given by the discrete choice probabilities (27), can be simplified by noting that they are not affected if a term that is independent of $h$ is subtracted from all fitnesses $U_{h, t-1}$. Therefore, we can ignore the term $\left(-R x_{t-1}+a \sigma^{2} z^{s}\right)\left(x_{t}-R x_{t-1}+a \sigma^{2} z^{s}\right)$ in the numerator of the second part of (40) to obtain the equivalent fitness

$$
U_{h, t-1}=\left(x_{t-1}-R x_{t-2}+a \sigma^{2} z^{s}\right) \frac{f_{h, t-2}}{a \sigma^{2}} .
$$

Using (36), the LTL for linear forecasting rules (38) is then given by

$$
x_{t}=\frac{1}{R} \frac{E\left[f_{t}\left(\vartheta_{0}\right) e^{\eta\left(x_{t-1}-R x_{t-2}+a \sigma^{2} z^{s}\right) f_{t-2}\left(\vartheta_{0}\right)}\right]}{E\left[e^{\eta\left(x_{t-1}-R x_{t-2}+a \sigma^{2} z^{s}\right) f_{t-2}\left(\vartheta_{0}\right)}\right]},
$$

\footnotetext{
${ }^{7}$ In particular, with fitness as in (28) given by a weighted average of all past profits with exponentially decreasing weights, the LTL becomes infinite dimensional with infinitely many terms with exponentially decreasing weights added. When fitness is given by a weighted average of $L$ past profits, the dimension of the LTL becomes $L+d+1$.
} 
where $\eta \equiv \beta /\left(a \sigma^{2}\right)$ and

$$
f_{t}\left(\vartheta_{0}\right)=\vartheta_{00}+\vartheta_{01} x_{t-1}+\cdots+\vartheta_{0 d} x_{t-d}
$$

with $\vartheta_{0}=\left(\vartheta_{00}, \vartheta_{01}, \cdots, \vartheta_{0 d}\right)$ multivariate normal. Introduce $s_{0}=\eta\left(x_{t-1}-R x_{t-2}+a \sigma^{2} z^{s}\right)$, $s_{j}=\eta\left(x_{t-1}-R x_{t-2}+a \sigma^{2} z^{s}\right) x_{t-2-j}, 1 \leq j \leq d$ and put

$$
e^{\eta\left(x_{t-1}-R x_{t-2}+a \sigma^{2} z^{s}\right) f_{t-2}\left(\vartheta_{0}\right)}=\exp \left(\sum s_{j} \vartheta_{0 j}\right) .
$$

In order to calculate a closed form expression for the LTL (42) we use moment generating function formulae from normal distribution theory. Note that

$$
E\left\{\mathrm{e}^{N}\right\}=\exp \left(E\{N\}+\frac{1}{2} \operatorname{Var}\{N\}\right),
$$

for a normal random variable $N=\sum s_{j} \vartheta_{j}$. Note also that

$$
E\left[\vartheta_{0 k} \exp \left(\sum s_{j} \vartheta_{0 j}\right)\right]=\frac{d}{d s_{k}} E\left[\exp \left(\sum s_{j} \vartheta_{0 j}\right)\right] .
$$

Assume for simplicity that the $\vartheta_{j}$ are uncorrelated (equivalent to independence for this multivariate normal case). It is straightforward to extend the method to correlated $\vartheta_{j}$. Using the moment generating function formulae the following closed form expression for the LTL (42) is obtained:

$$
\begin{aligned}
R x_{t}= & \mu_{0}+\mu_{1} x_{t-1}+\ldots+\mu_{d} x_{t-d} \\
& +\eta\left(x_{t-1}-R x_{t-2}+a \sigma^{2} z^{s}\right)\left(\sigma_{0}^{2}+\sigma_{1}^{2} x_{t-1} x_{t-3}+\cdots+\sigma_{d}^{2} x_{t-d} x_{t-d-2}\right),
\end{aligned}
$$

where $\mu_{k}=E\left[\vartheta_{0 k}\right], \sigma_{k}^{2}=\operatorname{Var}\left[\vartheta_{0 k}\right], 0 \leq k \leq d$ and $\eta=\beta /\left(a \sigma^{2}\right)$.

Note that the fitness measure determines the actual distribution of the traders over the types, which is time-dependent; this distribution is given by

$$
\mathrm{d} \psi_{(\mathbf{x}, \lambda, \alpha)}=\frac{\mathrm{e}^{\beta U\left(\mathbf{x}, \lambda, \vartheta_{0}\right)}}{Z} \mathrm{~d} \nu_{\alpha}
$$

where $Z$ is a normalizing factor, $Z=\int \mathrm{d} \psi_{(\mathbf{x}, \lambda, \alpha)}$, and $\nu_{\alpha}$ is the initial distribution of beliefs at date 0 , as before. See also Diks and van der Weide (2002) for a recent extension of the LTLframework, where the measure $\mathrm{d} \psi$ is taken as the fundament of their Continuous Belief Systems. Since $\vartheta_{0}$ is multivariate normally distributed, and since $U$ is linear in $\vartheta_{0}$, the measure $\mathrm{d} \psi$ will be multivariate normal as well, with the same variances as $\mathrm{d} \nu_{\alpha}$, but with different means: if $E_{\psi} h(\vartheta)$ denotes $E h\left(\vartheta_{0}\right) \mathrm{e}^{\beta U} / E \mathrm{e}^{\beta U}$, then

$$
E_{\psi} \vartheta_{00}=\mu_{0}+\eta \sigma_{0}^{2}\left(x_{t-1}-R x_{t-2}\right), \quad E_{\psi} \vartheta_{0 j}=\mu_{j}+\eta \sigma_{j}^{2} x_{t-j-2}\left(x_{t-1}-R x_{t-2}\right) .
$$

These expressions show how the means of the forecasting coefficients $\vartheta_{0 j}$ of the population change over time. 
The simplest special case of (44) that still leads to interesting dynamics is obtained when all $\vartheta_{0 k}=0$, $1 \leq k \leq d$, that is, when the forecasting function (43) is purely biased: $f_{t}\left(\vartheta_{0}\right)=\vartheta_{00}$. In this case, the LTL reduces to the linear system

$$
R x_{t}=\mu_{0}+\eta \sigma_{0}^{2}\left(x_{t-1}-R x_{t-2}+a \sigma^{2} z^{s}\right)
$$

This simplest case already provides important economic intuition about the (in)stability of the (fundamental) steady state in an evolutionary system with many trader types. When there is no intrinsic mean bias (that is $\mu_{0}=0$ ), and the risk premium is zero $\left(z^{s}=0\right)$, the steady state of the LTL (46) coincides with the fundamental: $x^{*}=0$. When the mean bias and risk premium are both positive (negative) the steady state deviation $x^{*}$ will be positive (negative) so that the steady state will be above (below) the fundamental. The natural bifurcation parameter tuning the (in)stability of the system is $\eta \sigma_{0}^{2}=\beta \sigma_{0}^{2} / a \sigma^{2}$. We see that instability occurs if and only if $\eta$ increases beyond the bifurcation point $\eta_{c}=1 / \sigma_{0}^{2}$. Hence this simple case already suggests forces that may destabilize the evolutionary system: an increase in choice intensity $\beta$ for evolutionary selection, a decrease in risk aversion " $a$ ", a decrease in conditional variance of excess returns $\sigma^{2}$, or an increase in the diversity of purely biased beliefs $\sigma_{0}^{2}$. All of these can push $\kappa$ beyond $\kappa_{c}$, thereby triggering instability of the (fundamental) steady state.

The LTL (46), obtained for the simplest case where all trader types are purely biased, is a linear system, which is either globally stable or globally unstable. Except for the hairline case at the border of stability, $\eta=\eta_{c}$, bounded solutions not converging to the steady state do not exist in this simplest case. When lags are included in the linear forecasting rules, the LTL (44) becomes a nonlinear system exhibiting much richer dynamical behavior. We consider a simple but typical example, where the forecasting rules are taken again linear, but now with three lags:

$$
f_{t}\left(\vartheta_{0}\right)=\vartheta_{00}+\vartheta_{01} x_{t-1}+\vartheta_{02} x_{t-2}+\vartheta_{03} x_{t-3}
$$

The corresponding LTL is a 5-D nonlinear system:

$$
\begin{aligned}
R x_{t}= & \mu_{0}+\mu_{1} x_{t-1}+\mu_{2} x_{t-2}+\mu_{3} x_{t-3} \\
& +\eta\left(x_{t-1}-R x_{t-2}+a \sigma^{2} z^{s}\right)\left(\sigma_{0}^{2}+\sigma_{1}^{2} x_{t-1} x_{t-3}+\sigma_{2}^{2} x_{t-2} x_{t-4}+\sigma_{3}^{2} x_{t-3} x_{t-5}\right)
\end{aligned}
$$

where $\eta=\beta /\left(a \sigma^{2}\right)$ as before. We will discuss the most important generic and persistent features of the dynamical behavior of this 5-D LTL; higher dimensional versions of the LTL exhibit similar dynamical behavior.

[Figure 2 about here.] 
First we investigate steady states and their stability. Figure 2 shows a 2-D bifurcation diagram in the $\left(\eta, \mu_{1}\right)$ parameter plane, where $\mu_{1}$ represents the mean of the first order stochastic trend variable $\vartheta_{01}$ in the forecasting rule (47). Recall that $\mu_{0}$ is the mean of the $x_{t}$-independent term $\vartheta_{00}$ in the forecasting rule (47); it models the 'mean bias' of the trader type. When $\mu_{0}=0$ and $a \sigma^{2} z_{s}=0$ (expressing that the risk premium is also zero), the LTL is symmetric with respect to the fundamental steady state and the LTL is therefore non-generic with respect to the universe of 'all' differentiable dynamical systems. The bifurcation curves in the symmetric case (figure $2 a$ ) have been computed analytically, whereas the bifurcation curves in the asymmetric case (figure 2b) have been computed numerically, using the software package AUTO97; see e.g. Kuznetsov (1998) for an extensive mathematical treatment of bifurcation theory. In the symmetric case (figure 2a), for parameters taking values in the region enclosed by the Hopf, period doubling (PD) and pitchfork (PF) bifurcation curves, the fundamental steady state is unique and stable. As the parameters cross the PF curve, two additional non-fundamental steady states are created, one above and one below the fundamental. Other routes to instability occur when crossing the PD curve, where the fundamental steady state becomes unstable and a (stable) 2-cycle is created, or when crossing the Hopf curve, where the fundamental steady state becomes unstable and a (stable) invariant circle with periodic or quasi-periodic dynamics is created. The pitchfork bifurcation curve is non-generic (that is, non-typical) in the nonsymmetric case, and it only occurs generically in the symmetric case. When the symmetry is broken by a non-zero mean bias $\mu_{0} \neq 0$, as illustrated in figure $2 \mathrm{~b}$ for $\mu_{0}=-0.1$, the PF curve consequently disappears; in its place, there are two generic co-dimension one bifurcation curves, a Hopf and a saddle-node (SN) bifurcation curve. When crossing the SN curve from below, two additional steady states are created, one stable and one unstable. Notice that, as illustrated in figure $2 \mathrm{c}$, when the perturbation is small (in the figure $\mu_{0}=-0.1$ ), the SN and the Hopf curves are close to the PF and the Hopf curves in the symmetric case. In this sense the bifurcation diagram depends continuously on the parameters, and it is useful to consider the symmetric LTL as an "organizing" center to study bifurcation phenomena in the generic, non-symmetric LTL.

Most relevant from an economic point of view seems to be the situation that the mean $\mu_{1}$ of the first order coefficient $\vartheta_{01}$ in the forecasting rule (47) satisfies $0 \leq \mu_{1} \leq 1$. In that case, the (fundamental) steady state loses stability in a Hopf bifurcation as the structural parameter $\eta$ increases. Figure 3 illustrates the dynamical behavior of the LTL as the parameter $\eta$ further increases. After the Hopf bifurcation, for $1.1 \leq \eta \leq 1.5$ (figures 3a-c), periodic and quasi-periodic dynamics on a stable invariant circle occur. The stable invariant circle loses stability in a secondary (quasi-periodic) Hopf bifurcation, and for $\eta=1.51$ and $\eta=1.52$, (quasi-)periodic dynamics on a stable invariant torus occur. Recall the Newhouse-Ruelle-Takens phenomenon that $C^{2}$-close to a system with 
a quasi-periodic Hopf bifurcation systems with strange attractors exist. The numerical observation that a quasi-periodic Hopf bifurcation arises in our LTL, together with the LTL convergence theorem therefore suggests that our evolutionary systems with many trader types can exhibit strange attractors. Advanced textbook treatments describing many of these complicated dynamical phenomena are e.g. Guckenheimer and Holmes (1986), Palis and Takens (1993) and Arrowsmith and Place (1991). Figures 3f-g show the unstable manifold of a periodic saddle point; the curling shape of the unstable manifold suggest near homoclinic bifurcations and breaking up of the invariant torus into a strange attractor. Figures $3 \mathrm{~h}-\mathrm{m}$ show that increasing $\eta$ further leads to a bifurcation route to chaos and strange attractors culminating into a 'large' strange attractor for $\eta=1.6$. In particular, figure 3 presents numerical evidence of the occurrence of what Brock and Hommes (1997) called a rational route to randomness, that is, a bifurcation route to strange attractors as the intensity of choice to switch forecasting strategies increases. If such rational routes to randomness occur for the LTL, the LTL convergence theorem implies that in evolutionary systems with many trader types rational routes to randomness occur with high probability.

Figure 4 shows the corresponding evolution of the means of the forecasting coefficients $\vartheta_{0 j}$ of the population, as desribed in (45). As the parameter $\eta$ increases (e.g. because the intensity of choice $\beta$ increases) the amplitude of the fluctuations of the mean forecasting coefficients increases and the fluctuations become more irregular.

[Figure 3 about here.]

[Figure 4 about here.]

[Figure 5 about here.]

\section{Concluding Remarks and Future Research}

We have presented a general theoretical framework for heterogneous market dynamics with many different trader types. Our notion of Large Type Limit (LTL) describes the dynamical behavior in a heterogeneous market with many competing trader types. All generic and persistent dynamical features, such as steady state, local bifurcations, (quasi-)periodic dynamics as well as homoclinic bifurcation routes to strange attractors occurring in an LTL, also occur with probability arbitrarily close to 1 in the corresponding heterogeneous market when the number of trader types is large.

We have applied the LTL framework to an evolutionary asset pricing model with heterogeneous beliefs belonging to any class of forecasting rules indexed by a finite dimensional vector designating 
the type. Within this theoretical framework, conditions can be obtained for which asset prices will reflect economic fundamentals as well as conditions leading to deviations from a RE benchmark fundamental and excess volatility. In particular, an increase in the "intensity of adaptation" to switch prediction strategies and an increase in the dispersion of potential belief types can lead to emergence of complicated dynamics for the trajectory of deviation from RE for asset returns. These dynamics are suggestive of complicated dynamics for volatility, and volume. The LTL convergence theorem shows that these phenomena are likely to arise in evolutionary systems with many trader types.

Our theoretical framework may be useful to address these questions also empirically. The RE fundamental benchmark is nested as a special case within the general model, and the present framework may thus be used to test whether the extra parameters are empirically relevant. See Baak (1999) and Chavas (2000) for empirical evidence of heterogeneity and the presence of non-rational traders in the hog and beef markets; see Manzan (2003) for empirical evidence that the rapid increase in stock prices in the late nineties has been reenforced by trend following trading rules. Our theoretical framework is also useful for experimental laboratory testing of the expectations hypothesis. By controlling the RE benchmark fundamental in the laboratory, it can be tested whether experimental markets will or will not deviate from the RE benchmark fundamental; see van de Velden (2001) and Hommes et al. (2002ab) for recent experimental results in this direction.

At this point it is useful to compare our results to some related literature in evolutionary finance. Recent work by Egenter, Lux, and Stauffer (1999), Yeh and Chen (2001), Challet and Marsili (2002), and Lux and Schornstein (2002) have shown in their models that the ability to mimic stylized facts in financial markets becomes difficult as the large system limit is taken (either numerically or theoretically). To put it another way, their work suggests that the ability of their models to mimic stylized facts of financial markets is a finite size effect: a finite number of agents appears to be necessary for the models to do a good job of mimicing the stylized facts. To put it yet another way, the large system limit dynamical system produced by their models generates time series behavior that does not look like behavior of financial time series (it is too "flat" or too "periodic"). The dynamics of the deterministic skeleton of an LTL system, as shown by the example LTL in subsection 4.3, may be quite complicated. A convenient feature of the LTL framework is that it has been written in deviations from a benchmark fundamental. In future work, it would be interesting to study the time series properties of LTL sysems buffeted with stochastic shocks (e.g. stochastic shocks in the supply of the risky asset) around a stochastic benchmark fundamental, and investigate whether the stylized facts of financial time series can be reproduced. Such an investigation is beyond the scope of this paper, but we briefly discuss how our work t relates to other models in the literature. There are major differences in the way the models are set up. Let us discuss first Challet and Marsili (2002), hereafter 
“CM”, then Lux and Schornstein (2002), and finally Egenter, Lux, and Stauffer (1999).

CM work in a generalized Minority Game (MG) context which is somewhat distant from the financial context of our model, but we can draw parallels with our work. Each of their $N$ agents is assigned a strategy at random from a strategy space which scales like $S=2^{P}$, where $P$ is a positive integer, and then agent $\mathrm{i}$ is not allowed to switch her strategy. She is allowed to stay out of the game or enter the game and "trade" (i.e. do "+1" or do " -1 " according to her strategy rule) using her randomly endowed strategy according to a discrete choice model. She scores if she makes an opposite choice to the majority (hence the name "minority game"). CM show that they get "realistic" volatility clustering for their measure of "returns", for games with a finite number of agents (not too large) but when they take a type of large system limit, where the size of the strategy space is scaled up as well as the number of agents, this property vanishes. It would require much more investigation outside the scope of this paper to explore the difference in behavior between our model and CM's adequately.

Lux and Schornstein (2002) work a genetic algorithm approach to agent-based modelling in the context of an exchange rate model with a continuum of equilibria due to Kareken and Wallace (1982). They give an explanation for the dependence of their results on the realism of their simulated exchange rates upon the number of agents. That is, the realism vanishes as the number of agents becomes very large. However, their exchange rate model has no "fundamental" to "anchor" the exchange rate whereas our model is strongly "anchored" by the fundamental when there is no trader heterogeneity. That is, our model has only one equilibrium when there is no trader heterogeneity, not a continuum. This difference makes it hard to compare the two models.

Egenter, Lux, and Stauffer (1999) simulate two stock market models and show that realism of the simulated returns series vanishes as the number of agents becomes very large. They remark upon similar or related phenomena showing up in some other market models and conclude that a model capable of generating realistic time series behavior must have a modest number of agent types. Their two models are closer in structure to ours. They conclude, "Thus, if these models are good description of markets, then real markets with their "chaotic" strong fluctuations are dominated by a rather limited number of large players." As we discuss further below, our "effective number" of traders may be rather modest due to the rather small dimensionality of our "characteristics space" from which sampling is done in our LTL limiting process.

Diks and van der Weide (2002) recently proposed an interesting extension of the LTL framework by introducing so-called Continuous Belief Systems (CBS). Recall that the starting point of the LTL approach is a continuum of traders, choosing their strategies from a large but finite number of strategy types; in the large type limit this number tends to infinity. Diks and van der Weide however consider 
a finite number of traders choosing their strategies from a continuum of types, whose distribution co-evolves with prices over time. When they take the number of agents to infinity, the CBS tends then to a deterministic system, which is in most cases identical to an LTL system. However, when the number of traders is finite, the CBS is a stochastic system, as the traders make stochastic errors in evaluating the performance of the different strategies. The CBS concept may therefore be useful to study the role of a "finite size" effect in heterogeneous agents modeling; Diks and van der Weide give an explicit discussion of the "effective number of traders" that is being felt by the market.

Aoki (2002) presents a model stimulated by statistical work on the "sampling of species problem" that leads to strong clustering on a few types. He models entry, exit, and strategy switching by traders and presents a result that suggests convergence to a rather small number of "clusters" which relates to the conclusion of Egenter, Lux, and Stauffer (1999) above on the necessity of a modest number of types to generate realistic time series behavior. The findings in Aoki's work suggests that it would be interesting to extend our work to allow entry and exit of traders. As we said above, one way to do it would be to add a reservation strategy to the logistic model where an agent just stays out of the market unless the best performance index of the available strategies exceeds a fixed number, $U_{0}$, say. This "strategy zero" could be added to our list $H$ of strategies and we could proceed much as in the present paper. This extension is beyond the scope of the current paper.

Our LTL method appears on the surface to generate opposite results for very large $H$, but a closer look at the finite diminsional character of characteristic space suggests that the "effective number" of our agents may be quite modest in size after all. This would certaintly be the case if the characteristic space from which we sampled was compact. Perhaps if we increased the dimensionality of our characteristics space as we increased $H$ we would obtain results like those of the authors discussed above. This fascinating question must be left to future research.

The concept of LTL developed in this paper is a general tool that can be used to enhance the tractability of models with large numbers of heterogeneous learning agents in many different contexts. While we have developed LTL theory in this paper in an illustrative financial model where beliefs differ only across conditional means of returns, the same idea can be applied to other modeling contexts. For example, consider models with heterogeneity in other dimensions besides beliefs on conditional means. An example of such work is Chiarella and He (2002). Their assumptions place a structure of beliefs on both conditional mean and conditional variance heterogeneity which is expressed in deviations form from the fundamental baseline. Hence their model appears especially amenable to LTL type analysis.

Another source of examples, is models with a hierarchy of dynamics from fast to slow where the heterogeneity of beliefs and learning is placed upon the slow dynamics. For an example of fast/slow 
dynamics, consider the paper of de Fontnouvelle (2000). Here the context is a standard noisy rational expectations asset pricing framework at the fast dynamical level. But agents in the model have a choice of purchasing an expensive more accurate signal (predictor) on the future earnings of the asset or using publically available information that costs nothing. Belief heterogeneity is placed upon the "slow hyperdynamics." Here agents must predict what fraction of their rivals purchase the expensive signal. LTL modeling as in this paper could be adapted to do that and help produce a model that is analytically tractable as we have shown here.

Another important issue in evolutionary market systems is whether some type, e.g. a rational fundamental trader, can drive out all other types. Evstigneev, Hens, and Schenk-Hoppé (2001), hereafter, “EHS” (2001), and Hens, Schenk-Hoppé, and Stalder (2002), hereafter, "HSS”, have generalized the well-known results of Blume and Easley (1992) to any complete or incomplete asset market. These authors show that investors who divide income proportionally to the expected relative returns of the assets will asymptotically accumulate the most wealth. Their main result is the discovery (actual the discovery of the rule was in an earlier paper by Hens and Schenk-Hoppé (2001)) of a simple and attractive rule that eventually garners all the wealth via market selection. To put it another way these authors show that strategy heterogeneity vanishes in the limit in their contexts. It is hard to relate this work to ours directly, because the model setups are different.

One way to compare is to conjecture following Brock and Hommes (1997, p. 11), that if memory $w$ in Equation (28) were set equal to one, and realized profits were replaced by "risk adjusted profits", an objective closely tied to the original objective (14) upon which the demands were based, then rational expectations would eventually drive out all the other strategies because relative losses would go to infinity for all strategies that deviated from rational expectations. If such a conjecture were true it would give us a comparison in the sense that one strategy drove out all the rest whereas in our current formulation, strategy heterogeneity persists forever.

Another potential way to compare would be to observe that our equation (13) gives the wealth dynamics if we use a given investment rule for $z_{t}$ of the form of HSS and EHS. Of course we would need more assets than just a risky asset and a bond with exogenous payout rate $R$ in order to make a direct comparison. But we could speculate that if multiple risky assets were introduced and the fitness measure of a strategy was accumulated wealth we could develop an LTL for a space of "rule types" of the proportional form of HSS. Notice that Blume and Easley (1992, p. 23) recall Samuelson's result that "simple" rules are "right" only for those investors who have logarithmic utility functions. This suggests that we might be able to develop an LTL theory that parallels HSS and EHS and even deliver a result like theirs that heterogeneity vanishes in the limit if the memory $w$ is one for appropriate "fitness" functions. All this is beyond the scope of the current paper. 
A trader's strategy may very much depend upon the objectives and time scales of investment decisions. These time scales in turn are shaped by different effective discount rates, temporal patterns of revenue inflows and outflows, different beliefs about possible nonstationarities at different time scales (e.g. Kurz (1997)), the different natures of the assets being traded, etc. Our LTL methodology should extend quite readily to any coevolutionary system provided that variables can be defined that enable one to write the resulting system in recursive dynamical system form on a finite dimensional state space. One can think of the stability results of EHS and HSS, that one strategy accumlates all wealth, as a result on long time scales. However, LeBaron (2002) has recently shown in the context of computationally-based evolutionary financial markets how difficult it may be for the more long term rational investor strategies to drive out "less rational" shorter term strategies if there are enough short term strategies present that are "moving" the market in a particular directions. Our LTL-framework may be useful to address this important issue. One could add strategies based upon EHR (2001), BE (1992), BH (1998), to a pool of other strategies including those based on LeBaron's (2002) simulations and let them compete against each other in an LTL setting. An important question then is whether, as the memory parameter $w$ in the fitness measure approached unity, rational expectations or fundamentalists would drive out all other belief systems. The memory parameters themselves could be drawn from an appropriate distribution as well as the predictor parameters and the same type of LTL analysis we did above could be used to formulate and prove theorems about the impact of time scale heterogeneity (captured in the dispersion of the distribution of memory parameters) upon the evolutionary stability of any particular strategy including one based upon EHS (2001). The interaction of time scale heterogeneity with the predictor heterogeneity that we studied above on overall evolutionary stability is likely to be very subtle indeed. Unfortunately these issues are beyond the scope of the current paper and we must leave all such extensions of the evolutionary LTL framework, as well as its experimental and empirical testing for future work. 


\section{References}

[1] Anderson, S., de Palma, A. and Thisse, J., (1993), Discrete choice theory of product differentiation, MIT Press, Cambridge.

[2] Aoki, M., (2002), Open Models of Share Markets With Two Dominant Types of Participants," Journal of Economic Behavior and Organization 49, 199-216.

[3] Arthur, W.B., Holland, J.H., LeBaron, B., Palmer, R. and Taylor, P., (1997) Asset pricing under endogenous expectations in an artificial stock market, In: Arthur, W., Lane, D. and Durlauf, S., (eds.) The economy as an evolving complex system II, Addison-Wesley.

[4] Arthur, W., Lane, D. and Durlauf, S., (eds.), (1997), The economy as an evolving complex system II, Addison-Wesley, Redwood City.

[5] Baak, S.J. (1999), Tests for bounded rationality with a linear dynamic model distorted by heterogeneous expectations, Journal of Economic Dynamics and Control 23, 1517-1543.

[6] Blume, L. and Easley, D., (1992), Evolution and Market Behavior, Journal of Economic Theory, 58, $9-40$.

[7] Boldrin, M. and Levine, D., (2001), Growth cycles and market crashes, Journal of Economic Theory 96, 13-39.

[8] Brock, W.A., (1993) Pathways to randomness in the economy: emergent nonlinearity and chaos in economics and finance, Estudios Económicos 8, 3-55.

[9] Brock, W.A., (1997), Asset Price Behavior in Complex Environments, in: Arthur, W.B., Durlauf, S.N., and Lane, D.A., eds., The Economy as an Evolving Complex System II, Addison-Wesley, Reading, MA, $385-423$.

[10] Brock, W.A., and de Fontnouvelle, P., (2000), Expectational Diversity in Monetary Economics, Journal of Economic Dynamics and Control 24, 725-759.

[11] Brock, W.A., and Hommes, C.H., (1997a) A rational route to randomness, Econometrica 65, 1059-1095.

[12] Brock, W.A., and Hommes, C.H., (1997b) Models of complexity in economics and finance, In: Hey, C. et al. (eds.), System Dynamics in Economic and Financial Models, Chapter 1, Wiley Publ., 3-41.

[13] Brock, W.A., and Hommes, C.H., (1998), Heterogeneous beliefs and routes to chaos in a simple asset pricing model, Journal of Economic Dynamics and Control 22, 1235-1274.

[14] Brock, W.A., and Hommes, C.H., (1999), Rational Animal Spirits, In: Herings, P.J.J., Laan, van der G. and Talman, A.J.J. eds., The Theory of Markets, North-Holland, Amsterdam, 109-137.

[15] Brock, W.A., Lakonishok, J. and LeBaron, B., (1992) Simple technical trading rules and the stochastic properties of stock returns, Journal of Finance 47, 1731-64.

[16] Brock, W.A. and LeBaron, (1996), A structural model for stock return volatility and trading volume, Review of Economics and Statistics 78, 94-110. 
[17] Broer, H.W., Huitema, G.B., Takens, F. and Braaksma, B.L.J., (1990) Unfoldings and bifurcations of quasi-periodic tori, Memoirs of the AMS 83, 1-175.

[18] Camerer, C., (1989), Bubbles and fads in asset prices, Journal of Economic Surveys 3, 3-41.

[19] Challet, D. and Marsili, M., (2002), Criticality and Finite Size Effects in a Simple Realistic Model of Stock Market, working paper Department of Theoretical Physics, Oxford University, INFM, TriesteSISSA Unit, Trieste Italy (http://xxx.lanl.gov/archive/cond-mat/0210549).

[20] Chavas, J.P., (2000) On information and market dynamics: the case of the U.S. beef market, Journal of Economic Dynamics and Control 24, 833-853.

[21] Chiarella, C., and He, X., (2000) Stability of competitive equilibria with heterogeneous beliefs and learning, Quantitative Finance Research Group, R.P. 37, University of Technology, Sydney.

[22] Chiarella, C., and He, X., (2002), Heterogeneous beliefs, risk and learning in a simple asset pricing model, Computational Economics 19, 95-132

[23] De Grauwe, P., Dewachter, H. and Embrechts, M. (1993), Exchange rate theory. Chaotic models of foreigh exchange markets, Blackwell, Oxford.

[24] De Long, J.B., Shleifer, A., Summers,L.H. and Waldmann, R.J., (1990) Noise trader risk in financial markets, Journal of Political Economy 98, 703-738.

[25] Diks, C. and van der Weide, R., (2002) Continuous belief dynamics, CeNDEF Working paper 02-11, University of Amsterdam.

[26] Egenter, E., Lux, T., Stauffer, D., (1999), Finite-size Effects in Monte Carlo Simulations of Two Stock Market Models, Physica A, 268, 250-256.

[27] Evans, G.W. and Honkapohja, S., (2001) Learning and expectations in macroeconomics, Princeton University Press, Princeton.

[28] Evstigneev, I., Hens, T., Schenk-Hoppé, R., (2001), Market Selection of Financial Trading Strategies: Global Stability, Mathematical Finance 12, 329-339.

[29] Farmer, J.D., (2002), Market force, ecology, and evolution, Industrial and Corporate Change 11, 895953.

[30] de Fontnouvelle, P., (2000), Information dynamics in financial markets, Macroeconomic Dynamics 4, 139-169.

[31] Frankel, J.A. and Froot, K.A., (1988) Chartists, Fundamentalists and the Demand for Dollars, Greek Economic Review 10, 49-102.

[32] Friedman, M., (1953) The case of flexible exchange rates, In: Essays in positive economics, Univ. Chicago Press.

[33] Gaunersdorfer, A., (2000) Endogenous fluctuations in a simple asset pricing model with heterogeneous beliefs, Journal of Economic Dynamics and Control 24, 799-831. 
[34] Gaunersdorfer, A. and Hommes, C.H., (2000), A nonlinear structural model for volatility clustering, CeNDEF working paper 00-02, University of Amsterdam.

[35] Gaunersdorfer, A., Hommes, C.H., and Wagener, F.O.O., (2000) Bifurcation routes to volatility clustering, CeNDEF working paper 00-04, University of Amsterdam.

[36] Grandmont, J.-M., (1998), Expectation formation and stability in large socio-economic systems, Econometrica $66,741-781$.

[37] Guckenheimer, J., Holmes, P., (1986), Nonlinear Oscillations, Dynamical Systems, and Bifurcations of Vector Fields, New York: Springer-Verlag.

[38] Hens, Th. and Schenk-Hoppé, R., Evolutionary Stability of Portfolio Rules in Incomplete Markets, Journal of Mathematical Economics, this issue

[39] Hens, Th., Schenk-Hoppé, R. and Stalder, M., (2002), An Application of Evolutionary Finance to Firms listed in the Swiss Market Index, Swiss Journal of Economics \& Statistics, 138, 465-488

[40] Hildenbrand, W., (1982), Core of an economy, In: Arrow, K., Intrilligator, M., eds., Handbook of Mathematical Economics Volume II, Amsterdam: North Holland, 831-877.

[41] Hirshleifer, (2001), Investor psychology and asset pricing, Journal of Finance 56, 1533-1597.

[42] Hommes, C.H., (2001), Financial markets as nonlinear adaptive evolutionary systems, Quantitative Finance 1, 149-167.

[43] Hommes, C.H., J. Sonnemans, J. Tuinstra and H. van de Velden, (2002a), Expectations and Bubbles in an Asset Pricing Experiments, CeNDEF Working Paper WP 02-05, University of Amsterdam.

[44] Hommes, C.H., J. Sonnemans, J. Tuinstra and H. van de Velden, (2002b), Coordination of Expectations in Asset Pricing Experiments, CeNDEF Working Paper, University of Amsterdam.

[45] Jennrich, R.I., (1969), Asymptotic properties of non-linear least squares estimators', Annals of Mathematical Statistics 40, 633-643.

[46] Kareken, J., and Wallace, N., (1982), On the Indeterminacy of Equilibrium Exchange Rates, Quarterly Journal of Economics 96, 207-222

[47] Keynes, J.M., (1936) The general theory of unemployment, interest and money, Harcourt, Brace and World.

[48] Kirman, A., (1981), Measure theory with applications to economics, In: Arrow, K., Intrilligator, M., eds., Handbook of Mathematical Economics Volume I, Amsterdam: North Holland, 159-209.

[49] Kirman, A., (1991) Epidemics of opinion and speculative bubbles in financial markets, In M. Taylor (ed.), Money and financial markets, Macmillan.

[50] Kirman, A. and Teyssière, G., (2000), Microeconomic models for long memory in the volatility of financial time series, working paper GREQAM DT 00A31, Marseille.

[51] Kleidon, A., (1994), Stock market crashes, In: Jarrow, K., Maksimovic, V. and Ziemba, W. (eds.), Finance Handbook, North Holland, Amsterdam. 
[52] Kurz, M., (ed.) (1997), Endogenous economic fluctuations, Springer Verlag, New York.

[53] Kuznetsov, Y.A. (1998), Elements of Applied Bifurcation Theory, 2-nd edition, Springer Verlag, Berlin.

[54] LeBaron, B., (2000), Agent based computational finance: suggested readings and early research, Journal of Economic Dynamics and Control 24, 679-702.

[55] LeBaron, B., (2002), Short-memory Traders and Their Impact on Group Learning in Financial Markets, Proceedings of the National Academy of Sciences (USA), Vol. 99, Suppl. 3, May 14, 7201-7206.

[56] LeBaron, B., Arthur, W.B. and Palmer, R. (1999) Time series properties of an artificial stock market, Journal of Economic Dynamics and Control 23, 1487-1516.

[57] Lux, T., (1995) Herd Behavior, Bubbles and Crashes, The Economic Journal 105, 881-896.

[58] Lux, T. and Marchesi, M. (1999) Scaling and criticality in a stochastic multi-agent model of a financial market, Nature Vol. 397, February 1999, 498-500.

[59] Lux, T. and Marchesi, M. (2000) Volatility clustering in financial markets: a micro-simulation of interacting agents, International Journal of Theoretical and Applied Finance 3, 675-702.

[60] Lux, T. and Schornstein, S., (2002), Genetic Learning as an Explanation of Stylized Facts of Foreign Exchange Markets, Department of Economics, University of Kiel, Germany and London School of Economics, London, England.

[61] Manski, C. and McFadden, D., (1981), Structural analysis of discrete data with econometric applications, MIT Press, Cambridge.

[62] Manzan, S. (2003), Essays in Nonlinear Economic Dynamics, Ph-D Thesis University of Amsterdam, Tinbergen Institute.

[63] Newhouse, S., Ruelle, D., and Takens, F. (1978), Occurrence of strange Axiom A attractors near quasiperiodic flows on $T^{m}, m \geq 3$, Communications in Mathematical Physics 64, 35-40.

[64] Palis, J. and Takens, F., (1993) Hyperbolicity \& sensitive chaotic dynamics at homoclinic bifurcations, Cambridge University Press.

[65] Plott, C. R. and Sunder, S., (1988), Rational expectations and the aggregation of diverse information in laboratory security markets, Econometrica 56, 1085-1118.

[66] Ruelle, D. and Takens, F., (1971) On the nature of turbulence, Communications in Mathematical Physics $20,167-192$.

[67] Sargent, T.J., (1993) Bounded rationality in macroeconomics, Clarendon Press, Oxford.

[68] Sargent, T.J., (1999) The Conquest of American Inflation, Princeton: Princeton University Press.

[69] Serfling, R., (1980), Approximation theorems of mathematical statistics, John Wiley and Sons, New York.

[70] Shiller, R.J., (1989) Market Volatility, MIT Press, Cambridge.

[71] Shiller, R.J., (2000), Irrational exuberance, Princeton University Press. 
[72] Shleifer, A., (2000) "Inefficient markets: An introduction to behavioral finance", Oxford University Press, Oxford.

[73] Smith, V., Suchanek, G.L. and Williams, A.W., (1988) Bubbles, crashes and endogenous expectations in experimental spot asset markets, Econometrica 56, 1119-51.

[74] Sobel, J., (2000), Economists' Models of Learning, Journal of Economic Theory 94, 241-261.

[75] Thaler, R., (1994) Quasi Rational Economics, Russel Sage Foundation.

[76] Vilder, de R.G., (1996), ”Complicated endogenous business cycles under gross substitutability”, Journal of Economic Theory 71, 416-442.

[77] Velden, van de, H., (2001), An experimental approach to expectation formation, Ph-D Thesis University of Amsterdam.

[78] Wang, J., (1994), A model of competitive stock trading volume, Journal of Political Economy 102, 127 168.

[79] Yeh, C.-H. and Chen, S.-H., (2001), The Influence of Market Size in an Artificial Stock Market: The Approach Based on Genetic Programming, Preprint: I-Shou University 

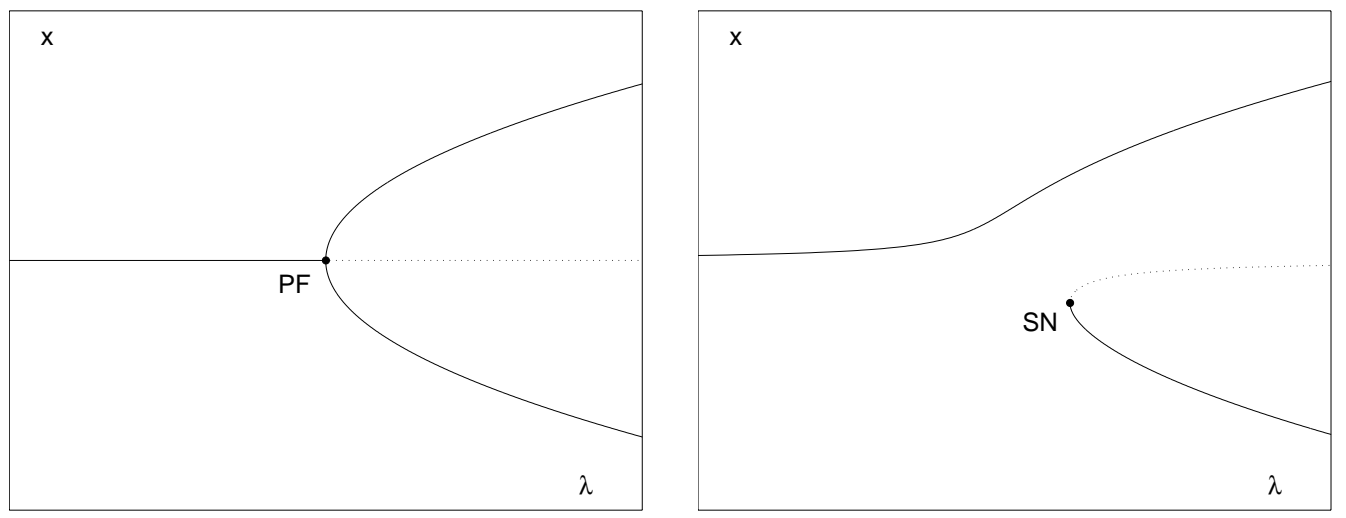

Figure 1: Bifurcation diagram for the pitchfork bifurcation (left panel) of $\Psi$ in (7) and the perturbed pitchfork bifurcation (right panel) in (8). In this $(\lambda, x)$-plot, the phase space is plotted vertically for every parameter $\lambda$. Drawn lines indicate the position of stable fixed points; dotted lines correspond to an unstable fixed point. The dot (PF) indicates the pitchfork bifurcation point. The pitchfork bifurcation does not persist. Note the occurrence of a saddle-node bifurcation (SN) in the perturbed map. The saddle-node bifurcation is in contrast to the pitchfork a generic co-dimension-1 bifurcation. 


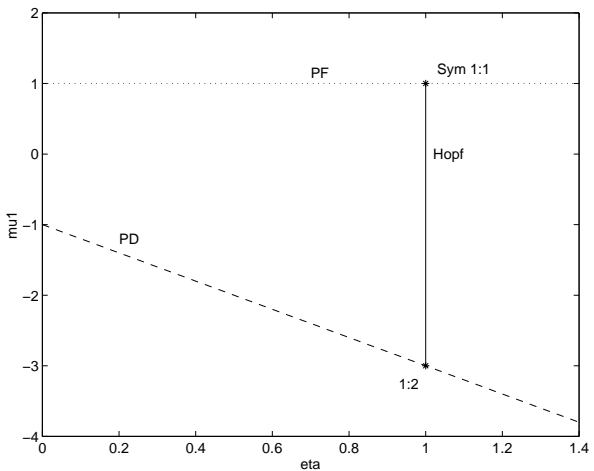

(a) symmetric case $\left(\mu_{0}=0\right)$

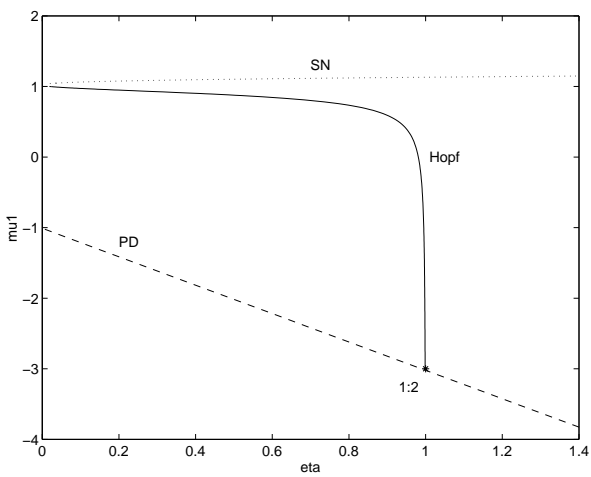

(b) generic, non-symmetric case $\left(\mu_{0}=-0.1\right)$

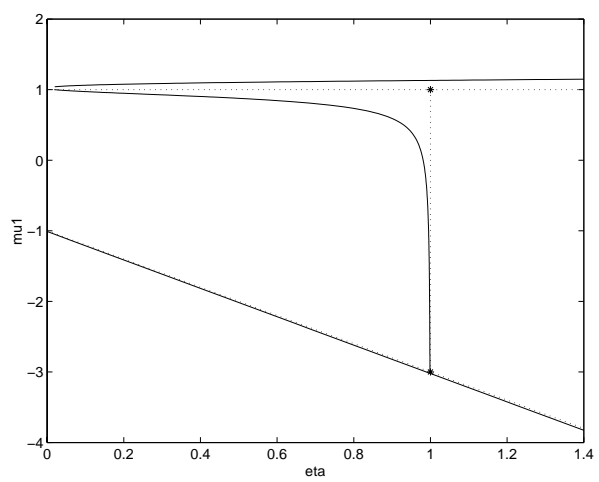

(c) non-symmetric and symmetric cases

Figure 2: Bifurcation diagrams in the $\left(\eta, \mu_{1}\right)$ parameter plane for $L T L(48)$, where $\mu_{1}$ represents the mean of the first order stochastic trend variable $\vartheta_{01}$ in the forecasting rule (47). For $\mu_{0}=a \sigma^{2}=0$, with $\mu_{0}$ the mean of the constant $\vartheta_{00}$ in the forecasting rule (47), the LTL is symmetric and thus non-generic; when $\mu_{0} \neq 0$ the LTL is non-symmetric and generic. The diagrams show Hopf $(H)$, period doubling $(P D)$, pitchfork $(P F)$ and saddle-node $(S N)$ bifurcation curves in $\left(\eta, \mu_{1}\right)$ parameter plane, with other parameters fixed at $R=1.01, z^{s}=0, \mu_{2}=\mu_{3}=0, \sigma_{0}=\sigma_{1}=\sigma_{2}=1$ and $\sigma_{3}=0$. Between the Hopf and PD curves (and the PF curve when $\mu_{0}=0$ ) there is a unique, stable steady state. This steady state becomes unstable when crossing the Hopf or the PD curve. Above the $P F$ curve or the $S N$ curve the system has three steady states. The PF curve is non-generic and only arises in the symmetric case with mean bias $\mu_{0}=0$. When the symmetry is broken by perturbing the mean bias to $\mu_{0}=-0.1$, the PF curve 'breaks' into generic Hopf and SN curves. 


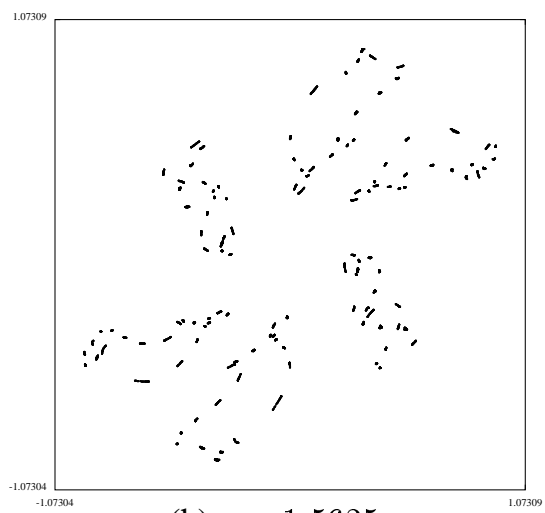

(h) $\eta=1.5625$

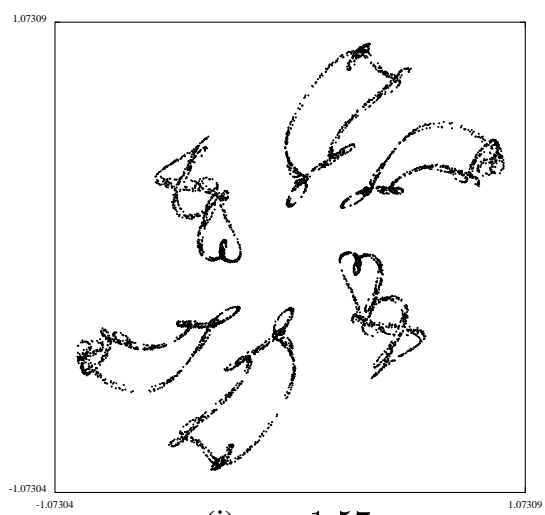

(j) $\eta=1.57$

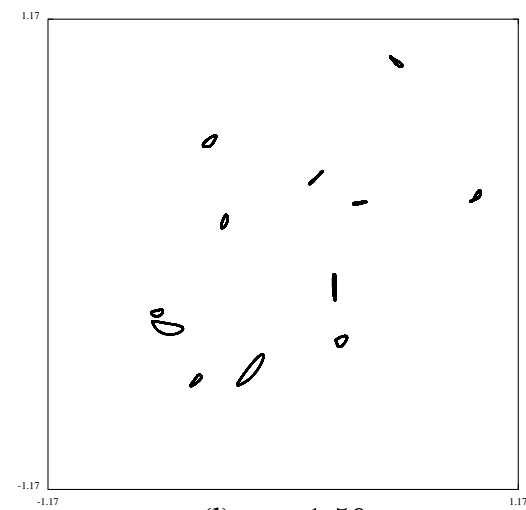

(1) $\eta=1.59$

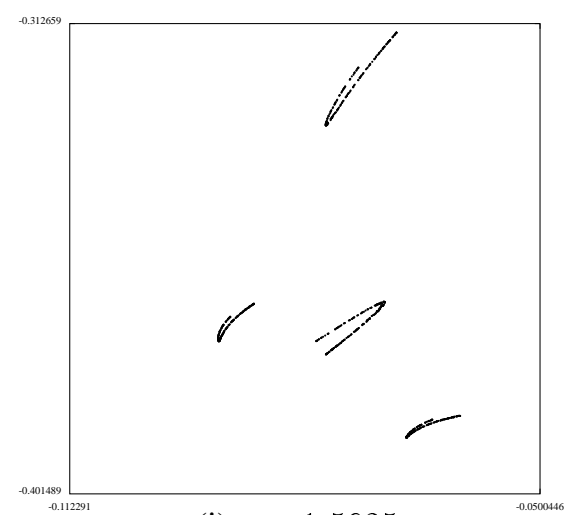

(i) $\eta=1.5625$

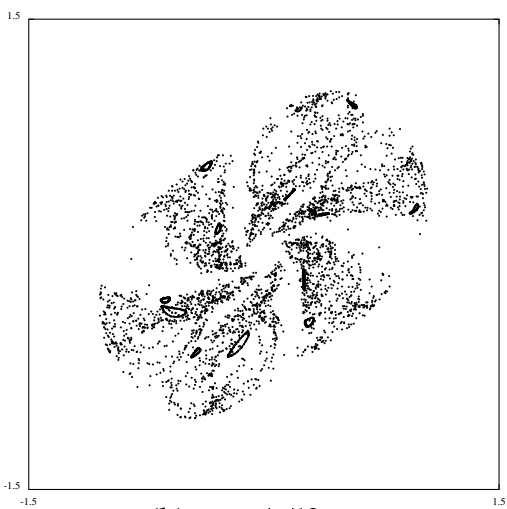

(k) $\eta=1.59$

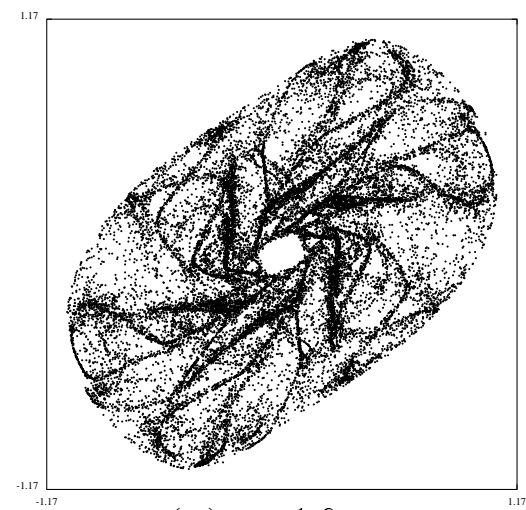

(m) $\eta=1.6$

Figure 3: Attractors in the phase space for the 5-D LTL with parameters $R=1.01, z^{s}=0, \mu_{0}=0$, $\mu_{1}=0, \mu_{2}=\mu_{3}=0, \sigma_{0}=\sigma_{1}=\sigma_{2}=\sigma_{3}=1$ : (a-c) immediately after the Hopf bifurcation periodic or quasi-periodic dynamics on a stable invariant circle occurs; (d-e) after a quasi-periodic Hopf bifurcation (quasi-)periodic dynamics on a stable invariant torus occurs; $(f-g)$ the unstable manifold of a periodic saddle point; the curling shape of the unstable manifold suggest near homoclinic bifurcations and breaking up of the invariant torus into a strange attractor; $(h-m)$ bifurcation route to chaos and strange attractors culminating into a 'large' strange attractor for $\eta=1.6$; note that in $(k)$ the transient dynamics are plotted for $\eta=1.59$, which look for a long time like the $d y$ namics of a chaotic attractor, before they settle down to the quasi-periodic long term behaviour seen in $(l)$. 

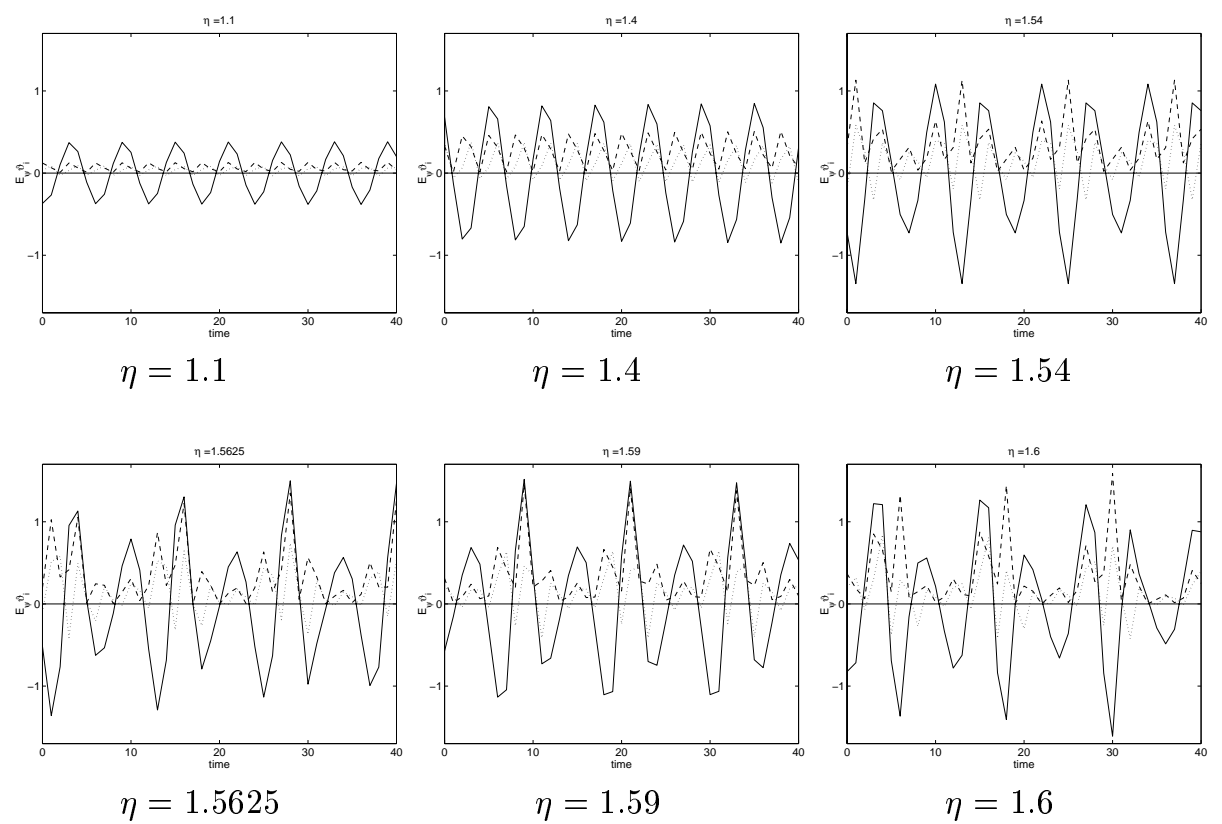

Figure 4: Dynamics of the large type limit distribution for the 5-D LTL with parameters $R=1.01$, $z^{s}=0, \mu_{0}=0, \mu_{1}=0, \mu_{2}=\mu_{3}=0, \sigma_{0}=\sigma_{1}=\sigma_{2}=\sigma_{3}=1$. Plotted are $E_{\psi} \vartheta_{00}$ (solid lines), $E_{\psi} \vartheta_{01}$ (dashed) and $E_{\psi} \vartheta_{02}$ (dotted). 